\title{
REALIZABILITY ALGEBRAS II : NEW MODELS OF ZF + DC
}

\author{
JEAN-LOUIS KRIVINE
}

Université Paris VII, C.N.R.S.

e-mail address: krivine@pps.univ-paris-diderot.fr

\begin{abstract}
Using the proof-program (Curry-Howard) correspondence, we give a new method to obtain models of $\mathrm{ZF}$ and relative consistency results in set theory. We show the relative consistency of $\mathrm{ZF}+\mathrm{DC}+$ there exists a sequence of subsets of $\mathrm{R}$ the cardinals of which are strictly decreasing + other similar properties of $\mathrm{R}$. These results seem not to have been previously obtained by forcing.
\end{abstract}

\section{INTRODUCTION}

The technology of classical realizability was developed in [15, 18, in order to extend the proof-program correspondence (also known as Curry-Howard correspondence) from pure intuitionistic logic to the whole of mathematical proofs, with excluded middle, axioms of $\mathrm{ZF}$, dependent choice, existence of a well ordering on $\mathcal{P}(\mathbb{N}), \ldots$

We show here that this technology is also a new method in order to build models of ZF and to obtain relative consistency results.

The main tools are :

- The notion of realizability algebra [18], which comes from combinatory logic [2] and plays a role similar to a set of forcing conditions. The extension from intuitionistic to classical logic was made possible by Griffin's discovery [7] of the relation between the law of Peirce and the instruction call-with-current-continuation of the programming language SCHEME. In this paper, we only use the simplest case of realizability algebra, which I call standard realizability algebra; somewhat like the binary tree in the case of forcing.

- The theory $\mathrm{ZF}_{\varepsilon}$ [13] which is a conservative extension of ZF, with a notion of strong membership, denoted as $\varepsilon$.

The theory $\mathrm{ZF}_{\varepsilon}$ is essentially $\mathrm{ZF}$ without the extensionality axiom. We note an analogy with the Fraenkel-Mostowski models with "urelements" : we obtain a non well orderable set, which is a Boolean algebra denoted $\mathbf{I 2}$, all elements of which (except 1) are empty. But we also notice two important differences :

1998 ACM Subject Classification: F.4.1.

Key words and phrases: Curry-Howard correspondence, set theory, combinatory logic, lambda-calculus, axiom of choice. 
- The final model of $\mathrm{ZF}+\neg \mathrm{AC}$ is obtained directly, without taking a suitable submodel.

- There exists an injection from the "pathological set" $\mathbf{I 2}$ into $\mathbb{R}$, and therefore $\mathbb{R}$ is also not well orderable.

We show the consistency, relatively to the consistency of ZF, of the theory ZF + DC (dependent choice) with the following properties:

there exists a sequence $\left(\mathcal{X}_{n}\right)_{n \in \mathbb{N}}$ of infinite subsets of $\mathbb{R}$, the "cardinals" of which are strictly increasing (this means that there is an injection but no surjection from $\mathcal{X}_{n}$ to $\mathcal{X}_{n+1}$ ), and such that $\mathcal{X}_{m} \times \mathcal{X}_{n}$ is equipotent with $\mathcal{X}_{m n}$ for $m, n \geq 2$;

there exists a sequence of infinite subsets of $\mathbb{R}$, the "cardinals" of which are strictly decreasing.

More detailed properties of $\mathbb{R}$ in this model are given in theorems 5.5 and 5.9 .

As far as I know, these consistency results are new, and it seems they cannot be obtained by forcing. But, in any case, the fact that the simplest non trivial realizability model (which I call the model of threads) has a real line with such unusual properties, is of interest in itself. Another aspect of these results, which is interesting from the point of view of computer science, is the following : in [18, we introduce read and write instructions in a global memory, in order to realize a weak form of the axiom of choice (well ordering of $\mathbb{R}$ ). Therefore, what we show here, is that these instructions are indispensable: without them, we can build a realizability model in which $\mathbb{R}$ is not well ordered.

\section{Standard ReAlizability ALGEBras}

The structure of realizability algebra, and the particular case of standard realizability algebra are defined in [18]. They are variants of the usual notion of combinatory algebra. Here, we only need the standard realizability algebras, the definition of which we recall below :

We have a countable set $\Pi_{0}$ which is the set of stack constants.

We define recursively two sets : $\Lambda$ (the set of terms) and $\Pi$ (the set of stacks). Terms and stacks are finite sequences of elements of the set :

which are obtained by the following rules :

$$
\Pi_{0} \cup\{B, C, E, I, K, W, \mathrm{cc}, \varsigma, \mathrm{k},(,),[,], \cdot\}
$$

- $B, C, E, I, K, W, \mathrm{cc}, \varsigma$ are terms (elementary combinators) ;

- each element of $\Pi_{0}$ is a stack (empty stacks) ;

- if $\xi, \eta$ are terms, then $(\xi) \eta$ is a term (this operation is called application);

- if $\xi$ is a term and $\pi$ a stack, then $\xi \cdot \pi$ is a stack (this operation is called push) ;

- if $\pi$ is a stack, then $\mathrm{k}[\pi]$ is a term.

A term of the form $\mathrm{k}[\pi]$ is called a continuation. From now on, it will be denoted as $\mathrm{k}_{\pi}$.

A term which does not contain any continuation (i.e. in which the symbol $k$ does not appear) is called proof-like.

Every stack has the form $\pi=\xi_{1} \cdot \ldots \cdot \xi_{n} \cdot \pi_{0}$, where $\xi_{1}, \ldots, \xi_{n} \in \Lambda$ and $\pi_{0} \in \Pi_{0}$, i.e. $\pi_{0}$ is a stack constant.

If $\xi \in \Lambda$ and $\pi \in \Pi$, the ordered pair $(\xi, \pi)$ is called a process and denoted as $\xi \star \pi$;

$\xi$ and $\pi$ are called respectively the head and the stack of the process $\xi \star \pi$.

The set of processes $\Lambda \times \Pi$ will also be written $\Lambda \star \Pi$. 


\section{Notation.}

For sake of brevity, the term $\left(\ldots\left(\left((\xi) \eta_{1}\right) \eta_{2}\right) \ldots\right) \eta_{n}$ will be also denoted as $(\xi) \eta_{1} \eta_{2} \ldots \eta_{n}$ or $\xi \eta_{1} \eta_{2} \ldots \eta_{n}$, if the meaning is clear. For example : $\xi \eta \zeta=(\xi) \eta \zeta=(\xi \eta) \zeta=((\xi) \eta) \zeta$.

We now choose a recursive bijection from $\Lambda$ onto $\mathbb{N}$, which is written $\xi \longmapsto \mathrm{n}_{\xi}$.

We put $\sigma=(B W)(B) B$ (the characteristic property of $\sigma$ is given below).

For each $n \in \mathbb{N}$, we define $\underline{n} \in \Lambda$ recursively, by putting: $\underline{0}=K I ; \underline{n+1}=(\sigma) \underline{n}$; $\underline{n}$ is the $n$-th integer and $\sigma$ is the successor in combinatory logic.

We define a preorder relation $\succ$ on $\Lambda \star \Pi$. It is the least reflexive and transitive relation such that, for all $\xi, \eta, \zeta \in \Lambda$ and $\pi, \varpi \in \Pi$, we have :

$(\xi) \eta \star \pi \succ \xi \star \eta \cdot \pi$.

$I \star \xi \cdot \pi \succ \xi \star \pi$.

$K \star \xi \cdot \eta \cdot \pi \succ \xi \star \pi$.

$E \star \xi \cdot \eta \cdot \pi \succ(\xi) \eta \star \pi$.

$W \star \xi \cdot \eta \cdot \pi \succ \xi \star \eta \cdot \eta \cdot \pi$.

$C \star \xi \cdot \eta \cdot \zeta \cdot \pi \succ \xi \star \zeta \cdot \eta \cdot \pi$.

$B \star \xi \cdot \eta \cdot \zeta \cdot \pi \succ(\xi)(\eta) \zeta \star \pi$.

$\mathrm{CC} \star \xi \cdot \pi \succ \xi \star \mathrm{k}_{\pi} \cdot \pi$.

$\mathrm{k}_{\pi} \star \xi \cdot \varpi \succ \xi \star \pi$.

$\varsigma \star \xi \cdot \eta \cdot \pi \succ \xi \star \underline{\mathrm{n}}_{\eta} \cdot \pi$.

For instance, with the definition of $\underline{0}$ and $\sigma$ given above, we have :

$\underline{0} \star \xi \cdot \eta \cdot \pi \succ \eta \star \pi ; \sigma \star \xi \cdot \eta \cdot \zeta \cdot \pi \succ(\xi \eta)(\eta) \zeta \star \pi$.

Finally, we have a subset $\Perp$ of $\Lambda \star \Pi$ which is a final segment for this preorder, which means

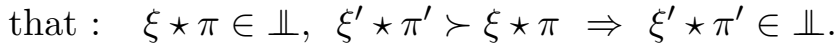

In other words, we ask that $\Perp$ has the following properties :

$(\xi) \eta \star \pi \notin \Perp \Perp \xi \star \eta \cdot \pi \notin \Perp \Perp$.

$I \star \xi \cdot \pi \notin \Perp \Rightarrow \xi \star \pi \notin \Perp$.

$K \star \xi \cdot \eta \cdot \pi \notin \Perp \Perp H \star \pi \notin \Perp \Perp$.

$E \star \xi \cdot \eta \cdot \pi \notin \Perp \Rightarrow(\xi) \eta \star \pi \notin \Perp$.

$W \star \xi \cdot \eta \cdot \pi \notin \Perp \Rightarrow \xi \star \eta \cdot \eta \cdot \pi \notin \Perp$.

$C \star \xi \cdot \eta \cdot \zeta \cdot \pi \notin \Perp \Perp \xi \star \zeta \cdot \eta \cdot \pi \notin \Perp \Perp$.

$B \star \xi \cdot \eta \cdot \zeta \cdot \pi \notin \Perp \Rightarrow(\xi)(\eta) \zeta \star \pi \notin \Perp \Perp$.

$\mathrm{cc} \star \xi \cdot \pi \notin \Perp \Rightarrow \xi \star \mathrm{k}_{\pi} \cdot \pi \notin \Perp$.

$\mathrm{k}_{\pi} \star \xi \cdot \varpi \notin \Perp \Rightarrow \xi \star \pi \notin \Perp$.

$\varsigma \star \xi \cdot \eta \cdot \pi \notin \Perp \Perp \xi \star \underline{\mathrm{n}}_{\eta} \cdot \pi \notin \Perp \Perp$.

Remark. Thus, the only arbitrary elements in a standard realizability algebra are the set $\Pi_{0}$ of stack constants and the set $\Perp$ of processes.

c-terms and $\lambda$-terms.

We call c-term a term which is built with variables, the elementary combinators $B, C, E$, $I, K, W, \mathrm{cc}, \varsigma$ and the application (binary function). A closed c-term is exactly what we have called a proof-like term.

Given a c-term $t$ and a variable $x$, we define inductively on $t$, a new c-term denoted by $\lambda x t$, which does not contain $x$. To this aim, we apply the first possible case in the following list :

1. $\lambda x t=(K) t$ if $t$ does not contain $x$.

2. $\lambda x x=I$. 
3. $\lambda x t u=(C \lambda x(E) t) u$ if $u$ does not contain $x$.

4. $\lambda x t x=(E) t$ if $t$ does not contain $x$.

5. $\lambda x t x=(W) \lambda x(E) t$ (if $t$ contains $x$ ).

6. $\lambda x(t)(u) v=\lambda x(B) t u v$ (if $u v$ contains $x$ ).

In [18, it is shown that this definition is correct. This allows us to translate every $\lambda$-term into a c-term. In the following, almost every c-term will be written as a $\lambda$-term. The fundamental property of this translation is given by theorem 1.1, which is proved in [18] :

Theorem 1.1. Let $t$ be a c-term with the only variables $x_{1}, \ldots, x_{n}$; let $\xi_{1}, \ldots, \xi_{n} \in \Lambda$ and $\pi \in \Pi$. Then $\lambda x_{1} \ldots \lambda x_{n} t \star \xi_{1} \cdot \ldots \cdot \xi_{n} \cdot \pi \succ t\left[\xi_{1} / x_{1}, \ldots, \xi_{n} / x_{n}\right] \star \pi$.

Remark. The property we need for the term $\sigma$ (the successor) is $\sigma \star \xi \cdot \eta \cdot \zeta \cdot \pi \succ(\xi \eta)(\eta) \zeta \star \pi$ (to prove theorem 4.12). Therefore, by theorem 1.1, we could define $\sigma=\lambda n \lambda f \lambda x(n f)(f) x$. The definition we chose is much simpler.

\section{THE FORMAL SYSTEM}

We write formulas and proofs in the language of first order logic. This formal language consists of :

- individual variables $x, y, \ldots$;

- function symbols $f, g, \ldots$; each one has an arity, which is an integer ; function symbols of arity 0 are called constant symbols.

- relation symbols; each one has an arity ; relation symbols of arity 0 are called propositional constants. We have two particular propositional constants $\top, \perp$ and three particular binary relation symbols $\notin, \notin, \subseteq$.

The terms are built in the usual way with individual variables and function symbols.

Remark. We use the word "term" with two different meanings : here as a term in a first order language, and previously as an element of the set $\Lambda$ of a realizability algebra. I think that, with the help of the context, no confusion is possible.

The atomic formulas are the expressions $R\left(t_{1}, \ldots, t_{n}\right)$, where $R$ is a $n$-ary relation symbol, and $t_{1}, \ldots, t_{n}$ are terms.

Formulas are built as usual, from atomic formulas, with the only logical symbols $\rightarrow, \forall$ :

- each atomic formula is a formula ;

- if $A, B$ are formulas, then $A \rightarrow B$ is a formula ;

- if $A$ is a formula and $x$ an individual variable, then $\forall x A$ is a formula.

Notations.

The formula $A_{1} \rightarrow\left(A_{2} \rightarrow\left(\cdots\left(A_{n} \rightarrow B\right) \cdots\right)\right)$ will be written $A_{1}, A_{2}, \ldots, A_{n} \rightarrow B$.

The usual logical symbols are defined as follows :

$\neg A \equiv A \rightarrow \perp ; A \vee B \equiv(A \rightarrow \perp),(B \rightarrow \perp) \rightarrow \perp ; A \wedge B \equiv(A, B \rightarrow \perp) \rightarrow \perp$;

$\exists x F \equiv \forall x(F \rightarrow \perp) \rightarrow \perp$.

More generally, we shall write $\exists x\left\{F_{1}, \ldots, F_{k}\right\}$ for $\forall x\left(F_{1}, \ldots, F_{k} \rightarrow \perp\right) \rightarrow \perp$.

We shall sometimes write $\vec{F}$ for a finite sequence of formulas $F_{1}, \ldots, F_{k}$;

Then, we shall also write $\vec{F} \rightarrow G$ for $F_{1}, \ldots, F_{k} \rightarrow G$ and $\exists x\{\vec{F}\}$ for $\forall x(\vec{F} \rightarrow \perp) \rightarrow \perp$. $A \leftrightarrow B$ is the pair of formulas $\{A \rightarrow B, B \rightarrow A\}$.

The rules of natural deduction are the following (the $A_{i}$ 's are formulas, the $x_{i}$ 's are variables of c-term, $t, u$ are c-terms, written as $\lambda$-terms) : 
1. $x_{1}: A_{1}, \ldots, x_{n}: A_{n} \vdash x_{i}: A_{i}$.

2. $x_{1}: A_{1}, \ldots, x_{n}: A_{n} \vdash t: A \rightarrow B, \quad x_{1}: A_{1}, \ldots, x_{n}: A_{n} \vdash u: A$

$$
\Rightarrow \quad x_{1}: A_{1}, \ldots, x_{n}: A_{n} \vdash t u: B .
$$

3. $x_{1}: A_{1}, \ldots, x_{n}: A_{n}, x: A \vdash t: B \Rightarrow x_{1}: A_{1}, \ldots, x_{n}: A_{n} \vdash \lambda x t: A \rightarrow B$.

4. $x_{1}: A_{1}, \ldots, x_{n}: A_{n} \vdash t: A \Rightarrow x_{1}: A_{1}, \ldots, x_{n}: A_{n} \vdash t: \forall x A$ where $x$ is an individual variable which does not appear in $A_{1}, \ldots, A_{n}$.

5. $x_{1}: A_{1}, \ldots, x_{n}: A_{n} \vdash t: \forall x A \Rightarrow x_{1}: A_{1}, \ldots, x_{n}: A_{n} \vdash t: A[\tau / x]$ where $x$ is an individual variable and $\tau$ is a term.

6. $x_{1}: A_{1}, \ldots, x_{n}: A_{n} \vdash \mathrm{cc}:((A \rightarrow B) \rightarrow A) \rightarrow A$ (law of Peirce).

7. $x_{1}: A_{1}, \ldots, x_{n}: A_{n} \vdash t: \perp \Rightarrow x_{1}: A_{1}, \ldots, x_{n}: A_{n} \vdash t: A$ for every formula $A$.

\section{THE THEORY $\mathrm{ZF}_{\varepsilon}$}

We write below a set of axioms for a theory called $\mathrm{ZF}_{\varepsilon}$. Then :

- We show that $\mathrm{ZF}_{\varepsilon}$ is a conservative extension of $\mathrm{ZF}$.

- We define the realizability models and we show that each axiom of $\mathrm{ZF}_{\varepsilon}$ is realized by a proof-like C-term, in every realizability model.

It follows that the axioms of $\mathrm{ZF}$ are also realized by proof-like c-terms in every realizability model.

We write the axioms of $\mathrm{ZF}_{\varepsilon}$ with the three binary relation symbols $\notin, \notin, \subseteq$. Of course, $x \varepsilon y$ and $x \in y$ are the formulas $x \notin y \rightarrow \perp$ and $x \notin y \rightarrow \perp$.

The notation $x \simeq y \rightarrow F$ means $x \subseteq y, y \subseteq x \rightarrow F$. Thus $x \simeq y$, which represents the usual (extensional) equality of sets, is the pair of formulas $\{x \subseteq y, y \subseteq x\}$.

We use the notations $(\forall x \varepsilon a) F(x)$ for $\forall x(\neg F(x) \rightarrow x \notin a)$ and

$(\exists x \varepsilon a) \vec{F}(x)$ for $\neg \forall x(\vec{F}(x) \rightarrow x \notin a)$.

For instance, $(\exists x \varepsilon y) t \simeq u$ is the formula $\neg \forall x(t \subseteq u, u \subseteq t \rightarrow x \notin y)$.

The axioms of $\mathrm{ZF}_{\varepsilon}$ are the following :

0 . Extensionality axioms.

$\forall x \forall y[x \in y \leftrightarrow(\exists z \varepsilon y) x \simeq z] ; \forall x \forall y[x \subseteq y \leftrightarrow(\forall z \varepsilon x) z \in y]$.

1. Foundation scheme.

$\forall x_{1} \ldots \forall x_{n} \forall a\left(\forall x\left((\forall y \varepsilon x) F\left[y, x_{1}, \ldots, x_{n}\right] \rightarrow F\left[x, x_{1}, \ldots, x_{n}\right]\right) \rightarrow F\left[a, x_{1}, \ldots, x_{n}\right]\right)$

for every formula $F\left[x, x_{1}, \ldots, x_{n}\right]$.

The intuitive meaning of axioms 0 and 1 is that $\varepsilon$ is a well founded relation, and that the relation $\in$ is obtained by "collapsing" $\varepsilon$ into an extensional binary relation.

The following axioms essentially express that the relation $\varepsilon$ satisfies the axioms of ZermeloFraenkel except extensionality.

2. Comprehension scheme.

$\forall x_{1} \ldots \forall x_{n} \forall a \exists b \forall x\left(x \varepsilon b \leftrightarrow\left(x \varepsilon a \wedge F\left[x, x_{1}, \ldots, x_{n}\right]\right)\right)$

for every formula $F\left[x, x_{1}, \ldots, x_{n}\right]$.

3. Pairing axiom.

$\forall a \forall b \exists x\{a \varepsilon x, b \varepsilon x\}$.

4. Union axiom.

$\forall a \exists b(\forall x \varepsilon a)(\forall y \varepsilon x) y \varepsilon b$.

5. Power set axiom.

$\forall a \exists b \forall x(\exists y \varepsilon b) \forall z(z \varepsilon y \leftrightarrow(z \varepsilon a \wedge z \varepsilon x))$. 
6. Collection scheme.

$\forall x_{1} \ldots \forall x_{n} \forall a \exists b(\forall x \varepsilon a)\left(\exists y F\left[x, y, x_{1}, \ldots, x_{n}\right] \rightarrow(\exists y \varepsilon b) F\left[x, y, x_{1}, \ldots, x_{n}\right]\right)$

for every formula $F\left[x, y, x_{1}, \ldots, x_{n}\right]$.

7. Infinity scheme.

$\forall x_{1} \ldots \forall x_{n} \forall a \exists b\left\{a \varepsilon b,(\forall x \varepsilon b)\left(\exists y F\left[x, y, x_{1}, \ldots, x_{n}\right] \rightarrow(\exists y \varepsilon b) F\left[x, y, x_{1}, \ldots, x_{n}\right]\right)\right\}$

for every formula $F\left[x, y, x_{1}, \ldots, x_{n}\right]$.

The usual Zermelo-Fraenkel set theory is obtained from $\mathrm{ZF}_{\varepsilon}$ by identifying the predicate symbols $\notin$ and $\notin$. Thus, the axioms of $\mathrm{ZF}$ are written as follows, with the predicate symbols $\notin, \subseteq($ recall that $x \simeq y$ is the conjunction of $x \subseteq y$ and $y \subseteq x)$ :

0 . Equality and extensionality axioms.

$\forall x \forall y[x \in y \leftrightarrow(\exists z \in y) x \simeq z] ; \forall x \forall y[x \subseteq y \leftrightarrow(\forall z \in x) z \in y]$.

1. Foundation scheme.

$\forall x_{1} \ldots \forall x_{n} \forall a\left(\forall x\left((\forall y \in x) F\left[y, x_{1}, \ldots, x_{n}\right] \rightarrow F\left[x, x_{1}, \ldots, x_{n}\right]\right) \rightarrow F\left[a, x_{1}, \ldots, x_{n}\right]\right)$

for every formula $F\left[x, x_{1}, \ldots, x_{n}\right]$ written with the only relation symbols $\notin, \subseteq$.

2. Comprehension scheme.

$\forall a \exists b \forall x\left(x \in b \leftrightarrow\left(x \in a \wedge F\left[x, x_{1}, \ldots, x_{n}\right]\right)\right)$

for every formula $F\left[x, x_{1}, \ldots, x_{n}\right]$ written with the only relation symbols $\notin, \subseteq$.

3. Pairing axiom.

$\forall a \forall b \exists x\{a \in x, b \in x\}$.

4. Union axiom.

$\forall a \exists b(\forall x \in a)(\forall y \in x) y \in b$.

5. Power set axiom.

$\forall a \exists b \forall x(\exists y \in b) \forall z(z \in y \leftrightarrow(z \in a \wedge z \in x))$.

6. Collection scheme.

$\forall x_{1} \ldots \forall x_{n} \forall a \exists b(\forall x \in a)\left(\exists y F\left[x, y, x_{1}, \ldots, x_{n}\right] \rightarrow(\exists y \in b) F\left[x, y, x_{1}, \ldots, x_{n}\right]\right)$

for every formula $F\left[x, y, x_{1}, \ldots, x_{n}\right]$ written with the only relation symbols $\notin, \subseteq$.

7. Infinity scheme.

$\forall x_{1} \ldots \forall x_{n} \forall a \exists b\left\{a \in b,(\forall x \in b)\left(\exists y F\left[x, y, x_{1}, \ldots, x_{n}\right] \rightarrow(\exists y \in b) F\left[x, y, x_{1}, \ldots, x_{n}\right]\right)\right\}$

for every formula $F\left[x, y, x_{1}, \ldots, x_{n}\right]$ written with the only relation symbols $\notin, \subseteq$.

Remark. The usual statement of the axiom of infinity is the particular case of this scheme, where $a$ is $\emptyset$, and $F(x, y)$ is the formula $y \simeq x \cup\{x\}$.

Let us show that $\mathrm{ZF}_{\varepsilon}$ is a conservative extension of $\mathrm{ZF}$. First, it is clear that, if $\mathrm{ZF}_{\varepsilon} \vdash F$, where $F$ is a formula of $\mathrm{ZF}$ (i.e. written only with $\notin$ and $\subseteq$ ), then $\mathrm{ZF} \vdash F$; indeed, it is sufficient to replace $\notin$ with $\notin$ in any proof of $\mathrm{ZF}_{\varepsilon} \vdash F$.

Conversely, we must show that each axiom of $\mathrm{ZF}$ is a consequence of $\mathrm{ZF}_{\varepsilon}$.

\section{Theorem 3.1.}

i) $Z F_{\varepsilon} \vdash \forall a(a \subseteq a)$ (and thus $a \simeq a$ ).

ii) $Z F_{\varepsilon} \vdash \forall a \forall x(x \varepsilon a \rightarrow x \in a)$.

Proof.

i) Using the foundation axiom, we assume $\forall x(x \varepsilon a \rightarrow x \subseteq x)$, and we must show $a \subseteq a$; therefore, we add the hypothesis $x \varepsilon a$. It follows that $x \subseteq x$, then $x \simeq x$, and therefore :

$\exists y\{x \simeq y, y \varepsilon a\}$, that is to say $x \in a$. Thus, we have $\forall x(x \varepsilon a \rightarrow x \in a)$, and therefore $a \subseteq a$.

ii) Just shown. 
Corollary 3.2. $Z F_{\varepsilon} \vdash \forall x(x \in a \rightarrow x \in b) \rightarrow a \subseteq b$.

Proof. We must show $x \varepsilon a \rightarrow x \in b$, which follows from $x \in a \rightarrow x \in b$ and $x \varepsilon a \rightarrow x \in a$ (theorem $3.1($ ii)).

Lemma 3.3. $Z F_{\varepsilon} \vdash a \subseteq b, \forall x(x \in b \rightarrow x \in c) \rightarrow a \subseteq c$.

Proof. We must show $x \varepsilon a \rightarrow x \in c$, which follows from $x \varepsilon a \rightarrow x \in b$ and $x \in b \rightarrow x \in c$.

Theorem 3.4. $Z F_{\varepsilon} \vdash \forall y \forall z(y \simeq a, a \in z \rightarrow y \in z) ; Z F_{\varepsilon} \vdash \forall y \forall z(a \subseteq y, z \in a \rightarrow z \in y)$.

Proof. Call $F(a), F^{\prime}(a)$ these two formulas. We show $F(a)$ by foundation :

thus, we suppose $(\forall x \varepsilon a) F(x)$ and we first show $F^{\prime}(a)$ : by hypothesis, we have $a \subseteq y$, $z \in a$; thus, there exists $a^{\prime}$ such that $z \simeq a^{\prime}$ and $a^{\prime} \varepsilon a$, and thus $F\left(a^{\prime}\right)$. From $a^{\prime} \varepsilon a$ and $a \subseteq y$, we deduce $a^{\prime} \in y$. From $z \simeq a^{\prime}$ and $a^{\prime} \in y$, we deduce $z \in y$ by $F\left(a^{\prime}\right)$.

Then, we show $F(a)$ : by hypothesis, we have $y \simeq a, a \in z$, thus $a \simeq y^{\prime}$ and $y^{\prime} \varepsilon z$ for some $y^{\prime}$. In order to show $y \in z$, it is sufficient to show $y \simeq y^{\prime}$.

Now, we have $y \simeq a, a \simeq y^{\prime}$, and thus $y^{\prime} \subseteq a, a \subseteq y$. From $F^{\prime}(a)$, we get $\forall z(z \in a \rightarrow z \in y)$; from $y^{\prime} \subseteq a$, we deduce $y^{\prime} \subseteq y$ by lemma 3.3 .

We have also $y \subseteq a, a \subseteq y^{\prime}$. From $F^{\prime}(a)$, we get $\forall z\left(z \in a \rightarrow z \in y^{\prime}\right)$; from $y \subseteq a$, we deduce $y \subseteq y^{\prime}$ by lemma 3.3 .

With corollary [3.2, we obtain :

Corollary 3.5. $Z F_{\varepsilon} \vdash b \subseteq c \leftrightarrow \forall x(x \in b \rightarrow x \in c)$.

It is now easy to deduce the equality and extensionality axioms of ZF :

$\forall x(x \simeq x) ; \forall x \forall y(x \simeq y \rightarrow y \simeq x) ; \forall x \forall y \forall z(x \simeq y, y \simeq z \rightarrow x \simeq z) ;$

$\forall x \forall x^{\prime} \forall y \forall y^{\prime}\left(x \simeq x^{\prime}, y \simeq y^{\prime}, x \notin y \rightarrow x^{\prime} \notin y^{\prime}\right) ; \forall x \forall y(\forall z(z \notin x \leftrightarrow z \notin y) \rightarrow x \simeq y) ;$

$\forall x \forall y(x \subseteq y \leftrightarrow \forall z(z \notin y \rightarrow z \notin x))$.

Remark. This shows that $\simeq$ is an equivalence relation which is compatible with the relations $\in$ and $\subseteq$; but, in general, it is not compatible with $\varepsilon$. It is the equality relation for ZF ; it will be called extensional equivalence.

Notation. The formula $\forall z(z \notin y \rightarrow z \notin x)$ will be written $x \subset y$. The ordered pair of formulas $x \subset y, y \subset x$ will be written $x \sim y$.

By theorem [3.1, we get $\mathrm{ZF}_{\varepsilon} \vdash \forall x \forall y(x \subset y \rightarrow x \subseteq y)$. Thus $\subset$ will be called strong inclusion, and $\sim$ will be called strong extensional equivalence.

- Foundation scheme.

Let $F[x]$ be written with only $\notin, \subseteq$ and let $G[x]$ be the formula $\forall y(y \simeq x \rightarrow F[y])$. Clearly, $\forall x G[x]$ is equivalent to $\forall x F[x]$. Therefore, from axiom scheme 1 of $\mathrm{ZF}_{\varepsilon}$, it is sufficient to show : $\forall b(\forall x(x \in b \rightarrow F[x]) \rightarrow F[b]) \rightarrow(\forall x(x \varepsilon a \rightarrow G[x]) \rightarrow G[a])$, i.e. :

$\forall b(\forall x(x \in b \rightarrow F[x]) \rightarrow F[b]), \forall x \forall y(x \varepsilon a, y \simeq x \rightarrow F[y]), a \simeq b \rightarrow F[b]$.

Therefore, it is sufficient to prove : $\forall x \forall y(x \varepsilon a, y \simeq x \rightarrow F[y]), a \simeq b \rightarrow \forall x(x \in b \rightarrow F[x])$. From $x \in b, a \simeq b$, we deduce $x \in a$ and therefore (by axiom 0 ), $x^{\prime} \varepsilon a$ for some $x^{\prime} \simeq x$. Finally, we get $F[x]$ from $\forall x \forall y(x \varepsilon a, y \simeq x \rightarrow F[y])$.

- Comprehension scheme : $\forall a \exists b \forall x(x \in b \leftrightarrow(x \in a \wedge F[x]))$

for every formula $F\left[x, x_{1}, \ldots, x_{n}\right]$ written with $\notin, \subseteq$.

From the axiom scheme 2 of $\mathrm{ZF}_{\varepsilon}$, we get $\forall x(x \varepsilon b \leftrightarrow(x \varepsilon a \wedge F[x]))$. If $x \in b$, then $x \simeq x^{\prime}$, $x^{\prime} \varepsilon b$ for some $x^{\prime}$. Thus $x^{\prime} \varepsilon a$ and $F\left[x^{\prime}\right]$. From $x \simeq x^{\prime}$ and $x^{\prime} \varepsilon a$, we deduce $x \in a$. Since $\subseteq$ and $\in$ are compatible with $\simeq$, it is the same for $F$; thus, we obtain $F[x]$. 
Conversely, if we have $F[x]$ and $x \in a$, we have $x \simeq x^{\prime}$ and $x^{\prime} \varepsilon a$ for some $x^{\prime}$. Since $F$ is compatible with $\simeq$, we get $F\left[x^{\prime}\right]$, thus $x^{\prime} \varepsilon b$ and $x \in b$.

- Pairing axiom : $\forall x \forall y \exists z\{x \in z, y \in z\}$.

Trivial consequence of axiom 3 of $\mathrm{ZF}_{\varepsilon}$, and theorem 3.1(ii).

- Union axiom : $\forall a \exists b \forall x \forall y(x \in a, y \in x \rightarrow y \in b)$.

From $x \in a$ we have $x \simeq x^{\prime}$ and $x^{\prime} \varepsilon a$ for some $x^{\prime}$; we have $y \in x$, therefore $y \in x^{\prime}$, thus $y \simeq y^{\prime}$ and $y^{\prime} \varepsilon x^{\prime}$ for some $y^{\prime}$. From axiom 4 of $\mathrm{ZF}_{\varepsilon}, x^{\prime} \varepsilon a$ and $y^{\prime} \varepsilon x^{\prime}$, we get $y^{\prime} \varepsilon b$; therefore $y \in b$, by $y \simeq y^{\prime}$.

- Power set axiom : $\forall a \exists b \forall x \exists y\{y \in b, \forall z(z \in y \leftrightarrow(z \in a \wedge z \in x))\}$

Given $a$, we obtain $b$ by axiom 5 of $\mathrm{ZF}_{\varepsilon}$; given $x$, we define $x^{\prime}$ by the condition :

$\forall z\left(z \varepsilon x^{\prime} \leftrightarrow(z \varepsilon a \wedge z \in x)\right)$ (comprehension scheme of $\left.\mathrm{ZF}_{\varepsilon}\right)$. By definition of $b$, there exists $y \varepsilon b$ such that $\forall z\left(z \varepsilon y \leftrightarrow z \varepsilon a \wedge z \varepsilon x^{\prime}\right)$, and therefore $\forall z(z \varepsilon y \leftrightarrow z \varepsilon a \wedge z \in x)$.

It follows easily that $\forall z(z \in y \leftrightarrow z \in a \wedge z \in x)$.

- Collection scheme : $\forall a \exists b(\forall x \in a)(\exists y F[x, y] \rightarrow(\exists y \in b) F[x, y])$

for every formula $F\left[x, y, x_{1}, \ldots, x_{n}\right]$ written with the only relation symbols $\notin, \subseteq$.

From $x \in a$ and $\exists y F[x, y]$, we get $x \simeq x^{\prime}, x^{\prime} \varepsilon a$ for some $x^{\prime}$, and thus $\exists y F\left[x^{\prime}, y\right]$ since $F$ is compatible with $\simeq$. From axiom scheme 6 of $\mathrm{ZF}_{\varepsilon}$, we get $(\exists y \varepsilon b) F\left[x^{\prime}, y\right]$, and therefore $(\exists y \in b) F[x, y]$, by theorem 3.1 (ii), again because $F$ is compatible with $\simeq$.

- Infinity scheme : $\forall a \exists b\{a \in b,(\forall x \in b)(\exists y F[x, y] \rightarrow(\exists y \in b) F[x, y])\}$

for every formula $F\left[x, y, x_{1}, \ldots, x_{n}\right]$ written with the only relation symbols $\notin, \subseteq$.

Same proof.

\section{Realizability MOdels of $\mathrm{ZF}_{\varepsilon}$}

As usual in relative consistency proofs, we start with a model $\mathcal{M}$ of $\mathrm{ZFC}$, called the ground model or the standard model. In particular, the integers of $\mathcal{M}$ are called the standard integers.

The elements of $\mathcal{M}$ will be called individuals.

In the sequel, the model $\mathcal{M}$ will be our universe, which means that every notion we consider is defined in $\mathcal{M}$. In particular, the realizability algebra $(\Lambda, \Pi, \Perp)$ is an individual of $\mathcal{M}$.

We define a realizability model $\mathcal{N}$, with the same set of individuals as $\mathcal{M}$. But $\mathcal{N}$ is not a model in the usual sense, because its truth values are subsets of $\Pi$ instead of being 0 or 1 . Therefore, although $\mathcal{M}$ and $\mathcal{N}$ have the same domain (the quantifier $\forall x$ describes the same domain for both), the model $\mathcal{N}$ may (and will, in all non trivial cases) have much more individuals than $\mathcal{M}$, because it has individuals which are not named. In particular, it will have non standard integers.

Remark. This is a great difference between realizability and forcing models of ZF. In a forcing model, each individual is named in the ground model ; it follows that integers, and even ordinals, are not changed.

For each closed formula $F$ with parameters in $\mathcal{M}$, we define two truth values : $\|F\| \subseteq \Pi$ and $|F| \subseteq \Lambda$.

$|F|$ is defined immediately from $\|F\|$ as follows :

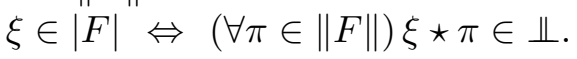

Notation. We shall write $\xi \Vdash F$ (read “ $\xi$ realizes $F$ ”) for $\xi \in|F|$. 
$\|F\|$ is now defined by recurrence on the length of $F$ :

- $F$ is atomic ;

then $F$ has one of the forms $\top, \perp, a \notin b, a \subseteq b, a \notin b$ where $a, b$ are parameters in $\mathcal{M}$.

We set :

$\|\top\|=\emptyset ; \quad\|\perp\|=\Pi ; \quad\|a \notin b\|=\{\pi \in \Pi ;(a, \pi) \in b\}$.

$\|a \subseteq b\|,\|a \notin b\|$ are defined simultaneously by induction on $(\operatorname{rk}(a) \cup \operatorname{rk}(b), \operatorname{rk}(a) \cap \operatorname{rk}(b))$

$(\operatorname{rk}(a)$ being the rank of $a)$.

$\|a \subseteq b\|=\bigcup_{c}\{\xi \cdot \pi ; \xi \in \Lambda, \pi \in \Pi,(c, \pi) \in a, \xi \Vdash c \notin b\} ;$

$\|a \notin b\|=\bigcup_{c}\left\{\xi \cdot \xi^{\prime} \cdot \pi ; \xi, \xi^{\prime} \in \Lambda, \pi \in \Pi,(c, \pi) \in b, \xi \Vdash a \subseteq c, \xi^{\prime} \Vdash c \subseteq a\right\}$.

- $F \equiv A \rightarrow B$; then $\|F\|=\{\xi \cdot \pi ; \xi\|A, \pi \in\| B \|\}$.

- $F \equiv \forall x A$ : then $\|F\|=\bigcup_{a}\|A[a / x]\|$.

The following theorem is an essential tool :

Theorem 4.1 (Adequacy lemma).

Let $A_{1}, \ldots, A_{n}, A$ be closed formulas of $Z F_{\varepsilon}$, and suppose that $x_{1}: A_{1}, \ldots, x_{n}: A_{n} \vdash t: A$. If $\xi_{1} \Vdash A_{1}, \ldots, \xi_{n} \Vdash A_{n}$ then $t\left[\xi_{1} / x_{1}, \ldots, \xi_{n} / x_{n}\right] \Vdash A$.

In particular, if $\vdash t: A$, then $t \Vdash A$.

We need to prove a (seemingly) more general result, that we state as a lemma :

Lemma 4.2. Let $A_{1}[\vec{z}], \ldots, A_{n}[\vec{z}], A[\vec{z}]$ be formulas of $Z F_{\varepsilon}$, with $\vec{z}=\left(z_{1}, \ldots, z_{k}\right)$ as free variables, and suppose that $x_{1}: A_{1}[\vec{z}], \ldots, x_{n}: A_{n}[\vec{z}] \vdash t: A[\vec{z}]$.

If $\xi_{1} \Vdash A_{1}[\vec{a}], \ldots, \xi_{n} \Vdash A_{n}[\vec{a}]$ for some parameters (i.e. individuals in $\mathcal{M}$ )

$\vec{a}=\left(a_{1}, \ldots, a_{k}\right)$, then $t\left[\xi_{1} / x_{1}, \ldots, \xi_{n} / x_{n}\right] \Vdash A[\vec{a}]$.

Proof. By recurrence on the length of the derivation of $x_{1}: A_{1}[\vec{z}], \ldots, x_{n}: A_{n}[\vec{z}] \vdash t: A[\vec{z}]$. We consider the last used rule.

1. $x_{1}: A_{1}[\vec{z}], \ldots, x_{n}: A_{n}[\vec{z}] \vdash x_{i}: A_{i}[\vec{z}]$. This case is trivial.

2. We have the hypotheses :

$x_{1}: A_{1}[\vec{z}], \ldots, x_{n}: A_{n}[\vec{z}] \vdash u: B[\vec{z}] \rightarrow A[\vec{z}] ; x_{1}: A_{1}[\vec{z}], \ldots, x_{n}: A_{n}[\vec{z}] \vdash v: B[\vec{z}] ; t=u v$.

By the induction hypothesis, we have $u[\vec{\xi} / \vec{x}] \Vdash B[\vec{a} / \vec{z}] \rightarrow A[\vec{a} / \vec{z}]$ and $v[\vec{\xi} / \vec{x}] \Vdash B[\vec{a} / \vec{z}]$.

Therefore $(u v)[\vec{\xi} / \vec{x}] \Vdash A[\vec{a} / \vec{z}]$ which is the desired result.

3. We have the hypotheses :

$x_{1}: A_{1}[\vec{z}], \ldots, x_{n}: A_{n}[\vec{z}], y: B[\vec{z}] \vdash u: C[\vec{z}] ; A[\vec{z}] \equiv B[\vec{z}] \rightarrow C[\vec{z}] ; t=\lambda y u$.

We want to show that $(\lambda y u)[\vec{\xi} / \vec{x}] \Vdash B[\vec{a} / \vec{z}] \rightarrow C[\vec{a} / \vec{z}]$. Thus, let :

$\eta \Vdash B[\vec{a} / \vec{z}]$ and $\pi \in\|C[\vec{a} / \vec{z}]\|$. We must show :

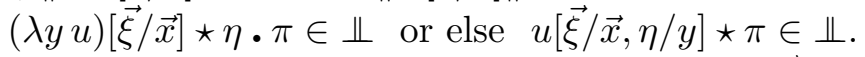

Now, by the induction hypothesis, we have $u[\vec{\xi} / \vec{x}, \eta / y] \Vdash C[\vec{a} / \vec{z}]$,

which gives the result.

4. We have the hypotheses:

$x_{1}: A_{1}[\vec{z}], \ldots, x_{n}: A_{n}[\vec{z}] \vdash t: B[\vec{z}] ; A[\vec{z}] \equiv \forall z_{1} B[\vec{z}] ; \xi_{i} \Vdash A_{i}\left[a_{1} / z_{1}, a_{2} / z_{2}, \ldots, a_{k} / z_{k}\right]$;

the variable $z_{1}$ is not free in $A_{1}[\vec{z}], \ldots, A_{n}[\vec{z}]$.

We have to show that $t[\vec{\xi} / \vec{x}] \Vdash \forall z_{1} B[\vec{a} / \vec{z}]$ i.e. $t[\vec{\xi} / \vec{x}] \Vdash \forall z_{1} B\left[a_{2} / z_{2}, \ldots, a_{k} / z_{k}\right]$. Thus, we 
take an arbitrary set $b$ in $\mathcal{M}$ and we show $t[\vec{\xi} / \vec{x}] \Vdash B\left[b / z_{1}, a_{2} / z_{2}, \ldots, a_{k} / z_{k}\right]$. By the induction hypothesis, it is sufficient to show that $\xi_{i} \Vdash A_{i}\left[b / z_{1}, a_{2} / z_{2}, \ldots, a_{k} / z_{k}\right]$. But this follows from the hypothesis on $\xi_{i}$, because $z_{1}$ is not free in the formulas $A_{i}$.

5. We have the hypotheses :

$x_{1}: A_{1}[\vec{z}], \ldots, x_{n}: A_{n}[\vec{z}] \vdash t: \forall y B[y, \vec{z}] ; A[\vec{z}] \equiv B[\tau[\vec{z}] / y, \vec{z}] ; \quad \xi_{i} \Vdash A_{i}[\vec{a}]$.

By the induction hypothesis, we have $t[\vec{\xi} / \vec{x}] \Vdash \forall y B[y, \vec{a} / \vec{z}]$; therefore $t[\vec{\xi} / \vec{x}] \Vdash B[b / y, \vec{a} / \vec{z}]$ for every parameter $b$. We get the desired result by taking $b=\tau[\vec{a}]$.

6. The result follows from the following :

Theorem 4.3. For every formulas $A, B$, we have cc $\Vdash((A \rightarrow B) \rightarrow A) \rightarrow A$.

Proof. Let $\xi \Vdash(A \rightarrow B) \rightarrow A$ and $\pi \in\|A\|$. Then $\mathrm{cc} \star \xi \bullet \pi \succ \xi \star \mathrm{k}_{\pi} \cdot \pi$ which is in $\Perp$, because $\mathrm{k}_{\pi} \Vdash A \rightarrow B$ by lemma 4.4

Lemma 4.4. If $\pi \in\|A\|$, then $k_{\pi} \Vdash A \rightarrow B$.

Proof. Indeed, let $\xi \Vdash A$; then $\mathrm{k}_{\pi} \star \xi \cdot \pi^{\prime} \succ \xi \star \pi \in \Perp$ for every stack $\pi^{\prime} \in\|B\|$.

7. We have the hypothesis $x_{1}: A_{1}[\vec{z}], \ldots, x_{n}: A_{n}[\vec{z}] \vdash t: \perp$.

By the induction hypothesis, we have $t[\vec{\xi} / \vec{x}] \| \perp$. Since $\|\perp\|=\Pi$, we have $t[\vec{\xi} / \vec{x}] \star \pi \in \Perp$ for every $\pi \in\|A[\vec{a} / \vec{z}]\|$, and therefore $t[\vec{\xi} / \vec{x}] \Vdash A[\vec{a} / \vec{z}]$ which is the desired result.

This completes the proof of lemma 4.2 and theorem 4.1.

Realized formulas and coherent models. In the ground model $\mathcal{M}$, we interpret the formulas of the language of $Z F$ : this language consists of $\notin, \subseteq$; we add some function symbols, but these functions are always defined, in $\mathcal{M}$, by some formulas written with $\notin, \subseteq$. We suppose that this ground model satisfies ZFC.

The value, in $\mathcal{M}$, of a closed formula $F$ of the language of $\mathrm{ZF}$, with parameters in $\mathcal{M}$, is of course 1 or 0 . In the first case, we say that $\mathcal{M}$ satisfies $F$, and we write $\mathcal{M} \models F$.

In the realizability model $\mathcal{N}$, we interpret the formulas of the language of $Z F_{\varepsilon}$, which consists of $\notin, \notin, \subseteq$ and the same function symbols as in the language of ZF. The domain of $\mathcal{N}$ and the interpretation of the function symbols are the same as for the model $\mathcal{M}$.

The value, in $\mathcal{N}$, of a closed formula $F$ of $\mathrm{ZF}_{\varepsilon}$ with parameters (in $\mathcal{M}$ or in $\mathcal{N}$, which is the same thing) is an element of $\mathcal{P}(\Pi)$ which is denoted as $\|F\|$, the definition of which has been given above.

Thus, we can no longer say that $\mathcal{N}$ satisfies (or not) a given closed formula $F$. But we shall say that $\mathcal{N}$ realizes $F$ (and we shall write $\mathcal{N} \Vdash F$ ), if there exists a proof-like term $\theta$ such that $\theta \Vdash F$. We say that two closed formulas $F, G$ are interchangeable if $\mathcal{N} \Vdash F \leftrightarrow G$.

Notice that, if $\|F\|=\|G\|$, then $F, G$ are interchangeable (indeed $I \Vdash F \rightarrow G$ ), but the converse is far from being true.

The model $\mathcal{N}$ allows us to make relative consistency proofs, since it is clear, from the adequacy lemma (theorem 4.1), that the class of formulas which are realized in $\mathcal{N}$ is closed by deduction in classical logic. Nevertheless, we must check that the realizability model $\mathcal{N}$ is coherent, i.e. that it does not realize the formula $\perp$. We can express this condition in the following form :

For every proof-like term $\theta$, there exists a stack $\pi \in \Pi$ such that $\theta \star \pi \notin \Perp$.

When the model $\mathcal{N}$ is coherent, it is not complete, except in trivial cases. This means that there exist closed formulas $F$ of $\mathrm{ZF}_{\varepsilon}$ such that $\mathcal{N} \| F$ and $\mathcal{N} \| \neg \neg$. 


\section{The axioms of $\mathrm{ZF}_{\varepsilon}$ are realized in $\mathcal{N}$.}

- Extensionality axioms.

We have $\|\forall z(z \notin b \rightarrow z \notin a)\|=\bigcup_{c}\{\xi \cdot \pi ; \xi \Vdash c \notin b, \pi \in\|c \notin a\|\}$

by definition of the value of $\|\forall z(z \notin b \rightarrow z \notin a)\|$;

and $\|a \subseteq b\|=\bigcup_{c}\{\xi \cdot \pi ;(c, \pi) \in a, \xi \Vdash c \notin b\}$ by definition of $\|a \subseteq b\|$.

Therefore, we have $\|a \subseteq b\|=\|\forall z(z \notin b \rightarrow z \notin a)\|$, so that :

$I \Vdash \forall x \forall y(x \subseteq y \rightarrow \forall z(z \notin y \rightarrow z \notin x))$ and $I \Vdash \forall x \forall y(\forall z(z \notin y \rightarrow z \notin x) \rightarrow x \subseteq y)$.

In the same way, we have :

$\|\forall z(a \subseteq z, z \subseteq a \rightarrow z \notin b)\|=\bigcup\left\{\xi \cdot \xi^{\prime} \cdot \pi ; \xi \Vdash a \subseteq c, \xi^{\prime} \Vdash c \subseteq a ; \pi \in\|c \notin b\|\right\}$

by definition of the value of $\stackrel{c}{\|} \forall z(a \subseteq z, z \subseteq a \rightarrow z \notin b) \|$;

and $\left.\|a \notin b\|=\bigcup_{c}\left\{\xi \cdot \xi^{\prime} \cdot \pi ;(c, \pi) \in b, \xi \Vdash a \subseteq c, \xi^{\prime} \Vdash c \subseteq a\right\}\right\}$ by definition of $\|a \notin b\|$.

Therefore, we have $\|a \notin b\|=\|\forall z(a \subseteq z, z \subseteq a \rightarrow z \notin b)\|$, so that :

$I \Vdash \forall x \forall y(x \notin y \rightarrow \forall z(x \subseteq z, z \subseteq x \rightarrow z \notin y)) ;$

$I \Vdash \forall x \forall y(\forall z(x \subseteq z, z \subseteq x \rightarrow z \notin y) \rightarrow x \notin y)$.

Notation. We shall write $\vec{\xi}$ for a finite sequence $\left(\xi_{1}, \ldots, \xi_{n}\right)$ of terms. Therefore, we shall write $\vec{\xi} \Vdash \vec{A}$ for $\xi_{i} \Vdash A_{i}(i=1, \ldots, n)$.

In particular, the notation $\vec{\xi} \Vdash a \simeq b$ means $\xi_{1} \Vdash a \subseteq b, \xi_{2} \Vdash b \subseteq a$;

the notation $\vec{\xi} \Vdash A \leftrightarrow B$ means $\xi_{1} \Vdash A \rightarrow B, \xi_{2} \Vdash B \rightarrow A$.

- Foundation scheme.

Theorem 4.5. For every finite sequence $\vec{F}\left[x, x_{1}, \ldots, x_{n}\right]$ of formulas, we have:

$\mathrm{Y} \Vdash \forall x(\forall y(\vec{F}[y] \rightarrow y \notin x), \vec{F}[x] \rightarrow \perp) \rightarrow \forall x(\vec{F}[x] \rightarrow \perp)$

with $\mathrm{Y}=A A$ and $A=\lambda a \lambda f(f)(a)$ af (Turing fixed point combinator).

Proof. Let $\xi \Vdash \forall x(\forall y(\vec{F}[y] \rightarrow y \notin x), \vec{F}[x] \rightarrow \perp)$. We show, by induction on the rank of $a$, that:

$\mathrm{Y} \star \xi \cdot \vec{\eta} \cdot \pi \in \Perp$, for every $\pi \in \Pi$ and $\vec{\eta} \Vdash \vec{F}[a]$.

Since $\mathrm{Y} \star \xi \cdot \vec{\eta} \cdot \pi \succ \xi \star \mathrm{Y} \xi \cdot \vec{\eta} \cdot \pi$, it suffices to show $\xi \star \mathrm{Y} \xi \cdot \vec{\eta} \cdot \pi \in \Perp$.

Now, $\quad \xi \Vdash \forall y(\vec{F}[y] \rightarrow y \notin a), \vec{F}[a] \rightarrow \perp$, so that it suffices to show $\mathrm{Y} \xi \Vdash \forall y(\vec{F}[y] \rightarrow y \notin a)$, in other words $\mathrm{Y} \xi \Vdash \vec{F}[b] \rightarrow b \notin a$ for every $b$. Let $\vec{\zeta} \Vdash \vec{F}[b]$ and $\varpi \in\|b \notin a\|$. Thus, we have $(b, \varpi) \in a$, therefore $\operatorname{rk}(b)<\operatorname{rk}(a)$ so that $Y \star \xi \cdot \vec{\zeta} \cdot \varpi \in \Perp$ by induction hypothesis. It follows that $Y \xi \star \vec{\zeta} \cdot \varpi \in \Perp$, which is the desired result.

It follows from theorem 4.5 that the axiom scheme 1 of $\mathrm{ZF}_{\varepsilon}$ (foundation) is realized.

- Comprehension scheme.

Let $a$ be a set, and $F[x]$ a formula with parameters. We put :

$b=\{(x, \xi \cdot \pi) ;(x, \pi) \in a, \xi \Vdash F[x]\}$; then, we have trivially $\|x \notin b\|=\|F(x) \rightarrow x \notin a\|$.

Therefore $I \Vdash \forall x(x \notin b \rightarrow(F(x) \rightarrow x \notin a))$ and $I \Vdash \forall x((F(x) \rightarrow x \notin a) \rightarrow x \notin b)$.

- Pairing axiom.

We consider two sets $a$ and $b$, and we put $c=\{a, b\} \times \Pi$. We have $\|a \notin c\|=\|b \notin c\|=\|\perp\|$, 
thus $I \Vdash a \varepsilon c$ and $I \Vdash b \varepsilon c$.

\section{Remark.}

Except in trivial cases, $c$ has many other elements than $a$ and $b$, which have no name in $\mathcal{M}$.

- Union axiom.

Given a set $a$, let $b=\mathrm{Cl}(a)$ (the transitive closure of $a$, i.e. the least transitive set which contains $a$ ). We show $\|y \notin b \rightarrow x \notin a\| \subseteq\|y \notin x \rightarrow x \notin a\|$ :

indeed, let $\xi \cdot \pi \in\|y \notin b \rightarrow x \notin a\|$, i.e. $\xi \Vdash y \notin b$ and $(x, \pi) \in a$.

Therefore, $x \subseteq \mathrm{Cl}(a)$, i.e. $x \subseteq b$ and thus $\|y \notin b\| \supset\|y \notin x\|$.

Thus, we have $\xi \Vdash y \notin x$, which gives the result.

It follows that $I \Vdash \forall x \forall y((y \notin x \rightarrow x \notin a) \rightarrow(y \notin b \rightarrow x \notin a))$.

- Power set axiom.

Given a set $a$, let $b=\mathcal{P}(\mathrm{Cl}(a) \times \Pi) \times \Pi$. For every set $x$, we put :

$y=\{(z, \xi \cdot \pi) ; \xi \Vdash z \varepsilon x,(z, \pi) \in a\}$. We have $y=\{(z, \xi \cdot \pi) ; \xi \Vdash z \varepsilon x, \pi \in\|z \notin a\|\}$, and therefore $\|z \notin y\|=\|z \varepsilon x \rightarrow z \notin a\|$. Thus :

$I \Vdash \forall z(z \notin y \rightarrow(z \varepsilon x \rightarrow z \notin a))$ and $I \Vdash \forall z((z \varepsilon x \rightarrow z \notin a) \rightarrow z \notin y)$.

Now, it is obvious that $y \in \mathcal{P}(\mathrm{Cl}(a) \times \Pi)$, and therefore $(y, \pi) \in b$ for every $\pi \in \Pi$.

Thus, we have $\|y \notin b\|=\Pi=\|\perp\|$. It follows that :

$\lambda f(f) I I \Vdash \forall x(\forall y(\forall z(z \notin y \rightarrow(z \varepsilon x \rightarrow z \notin a)), \forall z((z \varepsilon x \rightarrow z \notin a) \rightarrow z \notin y) \rightarrow y \notin b) \rightarrow \perp)$.

- Collection scheme.

Given a set $a$, and a formula $F[x, y]$ with parameters, let :

$b=\bigcup\{\Phi(x, \xi) \times \mathrm{Cl}(a) ; x \in \mathrm{Cl}(a), \xi \in \Lambda\}$ with

$\Phi(x, \xi)=\{y$ of minimum $\operatorname{rank} ; \xi \Vdash F[x, y]\}$ or $\Phi(x, \xi)=\emptyset$ if there is no such $y$.

We show that $\|\forall y(F[x, y] \rightarrow x \notin a)\| \subseteq\|\forall y(F[x, y] \rightarrow y \notin b)\|:$

Suppose indeed that $\xi \cdot \pi \in\|\forall y(F[x, y] \rightarrow x \notin a)\|$, i.e. $(x, \pi) \in a$ and $\xi \Vdash F[x, y]$ for some $y$. By definition of $\Phi(x, \xi)$, there exists $y^{\prime} \in \Phi(x, \xi)$. Moreover, we have :

$x \in \mathrm{Cl}(a), \pi \in \mathrm{Cl}(a)$, and therefore $\left(y^{\prime}, \pi\right) \in b$; it follows that $\pi \in\left\|y^{\prime} \notin b\right\|$. But, since $y^{\prime} \in \Phi(x, \xi)$, we have $\xi \Vdash F\left[x, y^{\prime}\right]$ and thus $\xi \cdot \pi \in\left\|F\left[x, y^{\prime}\right] \rightarrow y^{\prime} \notin b\right\|$, which gives the result. We have proved that $I \Vdash \forall x(\forall y(F[x, y] \rightarrow y \notin b) \rightarrow \forall y(F[x, y] \rightarrow x \notin a))$.

- Infinity scheme.

Given a set $a$, we define $b$ as the least set such that :

$$
\{a\} \times \Pi \subseteq b \text { and } \forall x(\forall \pi \in \Pi)(\forall \xi \in \Lambda)((x, \pi) \in b \Rightarrow \Phi(x, \xi) \times\{\pi\} \subseteq b)
$$

where $\Phi(x, \xi)$ is defined as above.

We have $\{a\} \times \Pi \subseteq b$, thus $\|a \notin b\|=\|\perp\|$, and therefore $I \Vdash a \varepsilon b$.

We now show that $\|\forall y(F[x, y] \rightarrow x \notin b)\| \subseteq\|\forall y(F[x, y] \rightarrow y \notin b)\|:$

Suppose indeed that $\xi \cdot \pi \in\|\forall y(F[x, y] \rightarrow x \notin b)\|$, i.e. $(x, \pi) \in b$ and $\xi \Vdash F[x, y]$ for some $y$. By definition of $\Phi(x, \xi)$, there exists $y^{\prime} \in \Phi(x, \xi)$. By definition of $b$, we have $\left(y^{\prime}, \pi\right) \in b$, i.e. $\pi \in\left\|y^{\prime} \notin b\right\|$. Now, since $y^{\prime} \in \Phi(x, \xi)$, we have $\xi \Vdash F\left[x, y^{\prime}\right]$ and thus : $\xi \cdot \pi \in\left\|F\left[x, y^{\prime}\right] \rightarrow y^{\prime} \notin b\right\|$, which gives the result.

We have proved that $I \Vdash a \varepsilon b$ and $I \Vdash \forall x(\forall y(F[x, y] \rightarrow y \notin b) \rightarrow \forall y(F[x, y] \rightarrow x \notin b))$. 


\section{Function symbols and equality.}

According to our needs, we shall add to the language of $\mathrm{ZF}_{\varepsilon}$, some function symbols $f, g, \ldots$ of any arity. A $k$-ary function symbol $f$ will be interpreted, in the realizability model $\mathcal{N}$, by a functional relation, which is defined in the ground model $\mathcal{M}$ by a formula $F\left[x_{1}, \ldots, x_{k}, y\right]$ of ZF. Thus, we assume that $\mathcal{M} \models \forall x_{1} \ldots \forall x_{k} \exists$ ! $y F\left[x_{1}, \ldots, x_{k}, y\right]$

$\left(\exists ! y F[y]\right.$ is the conjunction of $\forall y \forall y^{\prime}\left(F[y], F\left[y^{\prime}\right] \rightarrow y=y^{\prime}\right)$ and $\exists y F[y]$ ).

The axiom schemes of $\mathrm{ZF}_{\varepsilon}$, written in the extended language, are still realized in the model $\mathcal{N}$, because the above proofs remain valid.

On the other hand, in order to make sure that the axiom schemes of ZF, which use a $k$-ary function symbol $f$, are still realized, one must check that this symbol is compatible with $\simeq$, i.e. that the following formula is realized in $\mathcal{N}$ :

$\forall x_{1} \ldots \forall x_{k}\left(x_{1} \simeq y_{1}, \ldots, x_{k} \simeq y_{k} \rightarrow f x_{1} \ldots x_{k} \simeq f y_{1} \ldots y_{k}\right)$.

We now add a new rule to build formulas of $\mathrm{ZF}_{\varepsilon}$ :

If $t, u$ are two terms and $F$ is a formula of $\mathrm{ZF}_{\varepsilon}$, then $t=u \hookrightarrow F$ is a formula of $\mathrm{ZF}_{\varepsilon}$.

The formula $t=u \hookrightarrow \perp$ is denoted $t \neq u$.

The formula $t \neq u \rightarrow \perp$, i.e. $(t=u \hookrightarrow \perp) \rightarrow \perp$ is denoted $t=u$.

The truth value of these new formulas is defined as follows, assuming that $t, u, F$ are closed, with parameters in $\mathcal{N}$ :

$\|t=u \hookrightarrow F\|=\emptyset$ if $t \neq u ;\|t=u \hookrightarrow F\|=\|F\|$ if $t=u$.

It follows that :

$\|t \neq u\|=\emptyset=\|\top\|$ if $t \neq u ;\|t \neq u\|=\Pi=\|\perp\|$ if $t=u$;

$\|t=u\|=\|\top \rightarrow \perp\|$ if $t \neq u ;\|t=u\|=\|\perp \rightarrow \perp\|$ if $t=u$.

Proposition 4.6 shows that $t=u \hookrightarrow F$ and $t=u \rightarrow F$ are interchangeable.

\section{Proposition 4.6.}

i) $\lambda x(x) I \Vdash(t=u \rightarrow F) \rightarrow(t=u \hookrightarrow F)$;

ii) $\lambda x \lambda y(\mathrm{cc}) \lambda k(y)(k) x \Vdash(t=u \hookrightarrow F), t=u \rightarrow F$.

Proof.

i) Let $\xi \| t=u \rightarrow F$ and $\pi \in\|t=u \hookrightarrow F\|$. Thus, we have $t=u$ and $\pi \in\|F\|$.

We must show $\lambda x(x) I \star \xi \bullet \pi \in \Perp$, that is $\xi \star I \bullet \pi \in \Perp$. This is immediate, by hypothesis on $\xi$, since $I \Vdash t=u$.

ii) Let $\xi \Vdash t=u \hookrightarrow F, \eta \Vdash t=u$ and $\pi \in\|F\|$. We must show that :

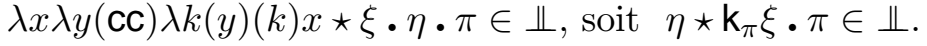

If $t \neq u$, then $\eta \Vdash \top \rightarrow \perp$, hence the result.

If $t=u$, then $\xi \Vdash F$, thus $\xi \star \pi \in \Perp$, therefore $\mathrm{k}_{\pi} \xi \Vdash \perp$.

But we have $\eta \Vdash \perp \rightarrow \perp$, and therefore $\eta \star \mathrm{k}_{\pi} \xi \cdot \pi \in \Perp$.

Proposition 4.7 shows that the formulas $t=u$ and $\forall x(u \notin x \rightarrow t \notin x)$ (Leibniz equality) are interchangeable.

\section{Proposition 4.7.}

i) $I \Vdash t=u \hookrightarrow \forall x(u \notin x \rightarrow t \notin x)$;

ii) $I \Vdash \forall x(u \notin x \rightarrow t \notin x) \rightarrow t=u$.

Proof.

i) It suffices to check that $I \Vdash \forall x(u \notin x \rightarrow t \notin x)$ when $t=u$, which is obvious.

ii) We must show that $I \Vdash \forall x(u \notin x \rightarrow t \notin x), t \neq u \rightarrow \perp$. Thus let $\xi \Vdash \forall x(u \notin x \rightarrow t \notin x)$, 
$\eta \Vdash t \neq u$ and $\pi \in \Pi$; we must show that $\xi \star \eta \cdot \pi \in \Perp$.

We have $\xi \Vdash u \notin a \rightarrow t \notin a$ for every $a$; we take $a=\{t\} \times \Pi$, thus $\|t \notin a\|=\Pi$, hence $\pi \in\|t \notin a\|$.

If $t=u$, we have $\eta \Vdash \perp$, thus $\eta \Vdash u \notin a$, hence the result.

If $t \neq u$, we have $\|u \notin a\|=\emptyset=\|\top\|$, thus $\eta \Vdash u \notin a$, hence the result.

We now show that the axioms of equality are realized.

Proposition 4.8. $I \Vdash \forall x(x=x) ; I \Vdash \forall x \forall y(x=y \hookrightarrow y=x)$;

$I \Vdash \forall x \forall y \forall z(x=y \hookrightarrow(y=z \hookrightarrow x=z))$;

$I \Vdash \forall x \forall y(x=y \hookrightarrow(F[x] \rightarrow F[y]))$ for every formula $F$ with one free variable, with parameters.

Proof. Trivial, by definition of $\hookrightarrow$.

Conservation of well-foundedness. Theorem 4.9 says that every well founded relation in the ground model $\mathcal{M}$, gives a well founded relation in the realizability model $\mathcal{N}$.

Theorem 4.9. Let $f$ be a binary function such that $f(x, y)=1$ is a well founded relation in the ground model $\mathcal{M}$. Then, for every formula $F[x]$ of $Z F_{\varepsilon}$ with parameters in $\mathcal{M}$ :

$\mathrm{Y} \Vdash \forall x(\forall y(f(y, x)=1 \hookrightarrow F[y]) \rightarrow F[x]) \rightarrow \forall x F[x]$

with $\mathrm{Y}=A A$ and $A=\lambda a \lambda f(f)(a) a f$.

Proof. Let us fix $a$ and let $\xi \Vdash \forall x(\forall y(f(y, x)=1 \hookrightarrow F[y]) \rightarrow F[x])$. We show, by induction on $a$, following the well founded relation $f(x, y)=1$, that $\mathrm{Y} \star \xi \bullet \pi \in \Perp$ for every $\pi \in\|F[a]\|$.

Thus, suppose that $\pi \in\|F[a]\|$; since $\mathrm{Y} \star \xi \cdot \pi \succ \xi \star \mathrm{Y} \xi \cdot \pi$, we need to show that $\xi \star \mathrm{Y} \xi \cdot \pi \in \Perp \Perp$. By hypothesis, we have $\xi \Vdash \forall y(f(y, a)=1 \hookrightarrow F[y]) \rightarrow F[a]$.

Thus, it suffices to show that $\mathrm{Y} \xi \Vdash f(y, a)=1 \hookrightarrow F[y]$ for every $y$.

This is clear if $f(y, a) \neq 1$, by definition of $\hookrightarrow$.

If $f(y, a)=1$, we must show $\mathrm{Y} \xi \Vdash F[y]$, i.e. $\mathrm{Y} \star \xi \bullet \rho \in \Perp$ for every $\rho \in\|F[y]\|$. But this follows from the induction hypothesis.

\section{Sets in $\mathcal{M}$ give type-like sets in $\mathcal{N}$.}

We define a unary function symbol I by putting $I(a)=a \times \Pi$ for every individual $a$ (element of the ground model $\mathcal{M}$ ).

For each set $E$ of the ground model $\mathcal{M}$, we also introduce the unary function $1_{E}$ with values in $\{0,1\}$, defined as follows :

$1_{E}(a)=1$ if $a \in E ; 1_{E}(a)=0$ if $a \notin E$.

The formula $1_{E}(x)=1 \hookrightarrow A$ will also be denoted as $x \varepsilon \beth E \hookrightarrow A$.

In particular, $a \notin\rfloor E$ is identical with $a \varepsilon \beth E \hookrightarrow \perp$ that is $1_{E}(a) \neq 1$.

We shall write $\forall x^{\beth E} A[x]$ for $\forall x(x \varepsilon \beth E \hookrightarrow A[x])$.

Proposition 4.6 shows that $x \in \Xi E \hookrightarrow A$ and $x \varepsilon \beth E \rightarrow A$ are interchangeable.

Therefore $\forall x^{\beth E} A[x]$ and $\forall x(x \varepsilon \beth E \rightarrow A[x])$ are also interchangeable. We have :

$$
\left\|\forall x^{\beth E} A[x]\right\|=\bigcup_{a \in E}\|A[a / x]\| \text { and }\left|\forall x^{\beth E} A[x]\right|=\bigcap_{a \in E}|A[a / x]| .
$$


As already said, we shall add to the language of $\mathrm{ZF}_{\varepsilon}$, some function symbols of any arity, which will be interpreted in the ground model $\mathcal{M}$ by some functional relations. Then every formula of the form $\forall \vec{x}\left(t_{1}[\vec{x}]=u_{1}[\vec{x}], \ldots, t_{k}[\vec{x}]=u_{k}[\vec{x}] \rightarrow t[\vec{x}]=u[\vec{x}]\right)$ which is satisfied in the model $\mathcal{M}$, is realized in the model $\mathcal{N}\left(t_{1}, u_{1}, \ldots, t_{k}, u_{k}, t, u\right.$ are terms of the language). Indeed, we verify immediately that :

$I \Vdash \forall \vec{x}\left(t_{1}[\vec{x}]=u_{1}[\vec{x}] \hookrightarrow\left(\ldots \hookrightarrow\left(t_{k}[\vec{x}]=u_{k}[\vec{x}] \hookrightarrow t[\vec{x}]=u[\vec{x}]\right)\right) \ldots\right)$.

It follows that if, for instance, $t\left[x_{0}, x_{1}\right]$ sends $E_{0} \times E_{1}$ into $D$ in the model $\mathcal{M}$, then it sends $\beth E_{0} \times I E_{1}$ into $I D$ in the model $\mathcal{N}$. Indeed, we have then :

$\mathcal{M} \models \forall x_{0} \forall x_{1}\left(1_{E_{0}}\left(x_{0}\right)=1,1_{E_{1}}\left(x_{1}\right)=1 \rightarrow 1_{D}\left(t\left[x_{0}, x_{1}\right]\right)=1\right)$ and therefore, we have :

$I \Vdash \forall x_{0} \forall x_{1}\left(1_{E_{0}}\left(x_{0}\right)=1 \hookrightarrow\left(1_{E_{1}}\left(x_{1}\right)=1 \hookrightarrow 1_{D}\left(t\left[x_{0}, x_{1}\right]\right)=1\right)\right)$, in other words :

$I \Vdash \forall x_{0}^{\beth E_{0}} \forall x_{1}^{\beth E_{1}}\left(t\left[x_{0}, x_{1}\right] \varepsilon \beth D\right)$.

Notice, in particular, that the characteristic function $1_{E}$, which takes its values in the set $\mathbf{2}=\{0,1\}$ in the model $\mathcal{M}$, sends $\beth E$ into $\mathbf{I} 2$ in the realizability model $\mathcal{N}$.

We shall denote $\wedge, \vee, \neg$ the (trivial) Boolean algebra operations in $\{0,1\}$ (they should not be confused with the logical connectives $\wedge, \vee, \neg$ ). In this way, we have defined three function symbols of the language of $\mathrm{ZF}_{\varepsilon}$; thus, in the realizability model $\mathcal{N}$, they define a Boolean algebra structure on the set $\mathbf{I 2}$.

\section{Remarks.}

i) A set of the form $I E$ behaves somewhat like a type, in the sense of computer science, because any function of the model $\mathcal{M}$ with domain (resp. range) $E_{1} \times \cdots \times E_{k}$ becomes a function of the model $\mathcal{N}$ with domain (resp. range) $I E_{1} \times \cdots \times I E_{k}$.

ii) The Boolean algebra $\mathbf{I 2}$ is, in general, non trivial i.e. it has $\varepsilon$-elements $\neq 0,1$. Notice that they are all empty : indeed, it is easy to check that $I \Vdash \forall x^{\mathrm{I2}} \forall y(x \neq 1 \rightarrow y \notin x)$.

\section{The set $\widetilde{\mathbb{N}}$ of integers in $\mathcal{N}$.}

We add to the language of $\mathrm{ZF}_{\varepsilon}$ a constant symbol 0 and a unary function symbol $s$. Their interpretation in the model $\mathcal{M}$ is as follows :

0 is $\emptyset ; s(a)$ is $\{a\} \times \Pi$ for every set $a$, in other words $s(a)=I(\{a\})$.

In the realizability model $\mathcal{N}, s(a)$ is the singleton of $a$. Indeed, we have trivially : $\|b \notin s(a)\|=\|b \neq a\|$ (i.e. $\emptyset$ if $a \neq b$ and $\Pi$ if $a=b$ ) and it follows that :

$I \Vdash \forall x \forall y(y \notin s x \rightarrow x \neq y) ; I \Vdash \forall x \forall y(x \neq y \rightarrow y \notin s x)$.

For each $n \in \mathbb{N}$, the term $s^{n} 0$ will also be written $n$.

Remark. In the definition of the set of integers in the realizability model $\mathcal{N}$, we prefer to use the singleton as the successor function $s$, instead of the usual one $x \longmapsto x \cup\{x\}$, which is more complicated to define. It would give : $s(a)=\{(a, K \bullet \pi) ; \pi \in \Pi\} \cup\{(x, \underline{0} \bullet \pi) ;(x, \pi) \in a\}$.

Theorem 4.10. The following formulas are realized in $\mathcal{N}$ :

i) $\forall x \forall y(s x=s y \hookrightarrow x=y)$;

ii) $\forall x(s x \not 0)$;

iii) $\forall x \forall y(x \simeq y \rightarrow s x \simeq s y)$;

iv) $\forall x \forall y(s x \simeq s y \rightarrow x \simeq y)$.

This shows, in particular, that the function $s$ is compatible with the extensional equivalence $\simeq$. 
Proof.

i) We check that $I \Vdash s a=s b \hookrightarrow a=b$. We may suppose $s a=s b$, because

$\|s a=s b \hookrightarrow a=b\|=\emptyset$ if $s a \neq s b$. But, in this case, we have $a=b$, by definition of $s a, s b$.

ii) We have $\|a \notin 0\|=\|\forall x(x \simeq a \rightarrow x \notin 0)\|=\emptyset$, since $\|x \notin 0\|=\emptyset$. Now $\|a \notin s a\|=\Pi$ and therefore we have, for any $\xi \in \Lambda, \lambda x(x) \xi \Vdash(a \notin \emptyset \rightarrow a \notin s a) \rightarrow \perp$; thus :

$\lambda x(x) \xi \Vdash \forall x(x \notin \emptyset \rightarrow x \notin s a) \rightarrow \perp$. But this means exactly that $\lambda x(x) \xi \Vdash s a \subseteq 0 \rightarrow \perp$, and therefore $\lambda x \lambda y(x) \xi \Vdash s a \simeq 0 \rightarrow \perp$.

iii) We show that the formula $a \simeq b \rightarrow s a \simeq s b$ is realized; it suffices to realize the formula $a \simeq b \rightarrow s a \subseteq s b$. We prove it by means of already realized sentences.

We need to prove $a \simeq b, x \notin s b \rightarrow x \notin s a$. But $x \notin s a$ has the same truth value as $x \neq a$. Thus, we simply have to prove $a \simeq b \rightarrow a \in s b$. But $a \in s b$ follows from $b \varepsilon s b$ and $a \simeq b$.

iv) In the same way, we prove the formula $s a \simeq s b \rightarrow a \simeq b$ and, in fact $s a \subseteq s b \rightarrow a \simeq b$. The formula $s a \subseteq s b$ is $\forall x(x \notin s b \rightarrow x \notin s a)$; but $x \notin s a$ is the same as $x \neq a$. Thus, from $s a \subseteq s b$ we obtain $a \in s b$, i.e. $(\exists x \varepsilon s b) x \simeq a$. But $x \varepsilon s b$ is the same as $x=b$, so that we obtain $a \simeq b$.

The individuals $s^{n} 0$ are obviously distinct, for $n \in \mathbb{N}$. Therefore, we can define :

$$
\widetilde{\mathbb{N}}=\left\{\left(s^{n} 0, \underline{n} \cdot \pi\right) ; n \in \mathbb{N}, \pi \in \Pi\right\}
$$

and we have :

$\|a \notin \widetilde{\mathbb{N}}\|=\emptyset$ if $a$ is not of the form $s^{n} 0$, with $n \in \mathbb{N}$;

$\left\|s^{n} 0 \notin \widetilde{\mathbb{N}}\right\|=\{\underline{n} \cdot \pi ; \pi \in \Pi\}$.

The formula $x \in \widetilde{\mathbb{N}}$ will also be written $\operatorname{ent}(x)$.

In the sequel, we shall use the restricted quantifier $\forall x^{\widetilde{\mathbb{N}}}$, which we also write $\forall x^{\mathrm{ent}}$, with the following meaning :

$\left\|\forall x^{\mathrm{ent}} F[x]\right\|=\left\|\forall x^{\widetilde{\mathbb{N}}} F[x]\right\|=\left\{\underline{n} \cdot \pi ; n \in \mathbb{N}, \pi \in\left\|F\left[s^{n} 0\right]\right\|\right\}$.

The restricted existential quantifier $\exists x^{\widetilde{\mathbb{N}}}$ or $\exists x^{\text {ent }}$ is defined as :

$\exists x^{\mathrm{ent}} F[x] \equiv \exists x^{\widetilde{\mathbb{N}}} F[x] \equiv \neg \forall x^{\mathrm{ent}} \neg F[x]$.

Proposition 4.11 shows that these quantifiers have indeed the intended meaning : the formulas $\forall x^{\text {ent }} F[x]$ and $\forall x(x \varepsilon \widetilde{\mathbb{N}} \rightarrow F[x])$ are interchangeable.

\section{Proposition 4.11.}

i) $\lambda x \lambda y \lambda z(y)(x) z \Vdash \forall x^{e n t} F[x] \rightarrow \forall x(\neg F[x] \rightarrow x \notin \widetilde{\mathbb{N}})$;

ii) $\lambda x \lambda y(\mathrm{cc}) \lambda k(x) k y \Vdash \forall x(\neg F[x] \rightarrow x \notin \widetilde{\mathbb{N}}) \rightarrow \forall x^{e n t} F[x]$.

Proof.

i) Let $\xi \Vdash \forall x^{\text {ent }} F[x], \eta \Vdash \neg F[a]$ and $\varpi \in\|a \notin \widetilde{\mathbb{N}}\|$. Thus, we have $a=s^{n} 0$ for some $n \in \mathbb{N}$ (else $\|a \notin \widetilde{\mathbb{N}}\|=\emptyset$ ) and $\varpi=\underline{n} \cdot \pi$. We must show that $\eta \star \xi \underline{n} \cdot \pi \in \Perp$.

Now, by hypothesis on $\xi$, we have $\xi \star \underline{n} \cdot \rho \in \Perp$ for any $\rho \in\left\|F\left[s^{n} 0\right]\right\|$; thus $\xi \underline{n} \Vdash F\left[s^{n} 0\right]$. Since $\eta \Vdash \neg F\left[s^{n} 0\right]$, we have $\eta \star \xi \underline{n} \cdot \pi \in \Perp \Perp \Perp$, which is the desired result.

ii) Let $\xi \| \forall x(\neg F[x] \rightarrow x \notin \widetilde{\mathbb{N}})$ and $\underline{n} \cdot \pi \in\left\|\forall x^{\text {ent }} F[x]\right\|$, with $n \in \mathbb{N}$ and $\pi \in\left\|F\left[s^{n} 0\right]\right\|$. We have : $\lambda x \lambda y(\mathrm{cc}) \lambda k(x) k y \star \xi \cdot \underline{n} \cdot \pi \succ \xi \star \mathrm{k}_{\pi} \cdot \underline{n} \cdot \pi$.

Now, we have $\mathrm{k}_{\pi} \Vdash \neg F\left[s^{n} 0\right]$ and $\underline{n} \cdot \pi \in\left\|s^{n} 0 \notin \widetilde{\mathbb{N}}\right\|$. Therefore $\xi \star \mathbf{k}_{\pi} \cdot \underline{n} \cdot \pi \in \Perp$.

Theorem 4.12 (Recurrence scheme). For every formula $F[\vec{x}, y]$ :

i) $I \Vdash \forall \vec{x} \forall n^{\widetilde{\mathbb{N}}}(\forall y(F[\vec{x}, s y] \rightarrow F[\vec{x}, y]), F[\vec{x}, n] \rightarrow F[\vec{x}, 0])$.

ii) $I \Vdash \forall \vec{x} \forall n^{\widetilde{\mathbb{N}}}(\forall y(F[\vec{x}, y] \rightarrow F[\vec{x}, s y]), F[\vec{x}, 0] \rightarrow F[\vec{x}, n])$. 
Proof.

i) Let $n \in \mathbb{N}, \quad \vec{a}$ a sequence of individuals, $\xi \Vdash \forall y(F[\vec{a}, s y] \rightarrow F[\vec{a}, y]), \pi \in\|F[\vec{a}, 0]\|$.

We must show that, for every $\alpha \Vdash F[\vec{a}, n]$, we have $I \star \underline{n} \cdot \xi \bullet \alpha \cdot \pi \in \Perp$.

In fact, we show, by recurrence on $n$, that $\underline{n} \star \xi \cdot \alpha \cdot \pi \in \Perp$.

This is immediate if $n=0$. In order to go from $n$ to $n+1$, we suppose now $\alpha \Vdash F[\vec{a}, s n]$; we have $\underline{n+1} \star \xi \cdot \alpha \cdot \pi \succ \sigma \underline{n} \star \xi \bullet \alpha \cdot \pi \succ \sigma \star \underline{n} \cdot \xi \bullet \alpha \cdot \pi \succ \underline{n} \star \xi \bullet \xi \alpha \cdot \pi$.

But, by hypothesis on $\xi$, we have $\xi \Vdash F[\vec{a}, s n] \rightarrow F[\vec{a}, n]$; thus $\xi \alpha \Vdash F[\vec{a}, n]$.

Hence the result, by the recurrence hypothesis.

ii) Let $n \in \mathbb{N}, \quad \vec{a}$ a sequence of individuals, $\xi \Vdash \forall y(F[\vec{a}, y] \rightarrow F[\vec{a}, s y]), \quad \alpha \Vdash F[\vec{a}, 0]$ and $\pi \in\|F[\vec{a}, 0]\|$. We must show that $I \star \underline{n} \cdot \xi \bullet \alpha \cdot \pi \in \Perp \Perp$; this follows from lemma 4.13, with $k=0$.

Lemma 4.13. Let $n, k \in \mathbb{N}, \quad \xi \Vdash \forall y(F[y] \rightarrow F[s y]), \quad \alpha \Vdash F\left[s^{k} 0\right]$ and $\pi \in\left\|F\left[s^{k} n\right]\right\|$. Then $\underline{n} \star \xi \cdot \alpha \cdot \pi \in \Perp \Perp \Perp$.

Proof. The proof is done for all integers $k$, by recurrence on $n$. This is immediate if $n=0$. In order to go from $n$ to $n+1$, we suppose now $\pi \in\left\|F\left[s^{k}(n+1)\right]\right\|$, i.e. $\pi \in\left\|F\left[s^{k+1} n\right]\right\|$. We have $\underline{n+1} \star \xi \cdot \alpha \cdot \pi \succ \sigma \underline{n} \star \xi \cdot \alpha \cdot \pi \succ \sigma \star \underline{n} \cdot \xi \bullet \alpha \cdot \pi \succ \underline{n} \star \xi \cdot \xi \alpha \cdot \pi$.

But, by hypothesis on $\xi$, we have $\xi \Vdash F\left[s^{k} 0\right] \rightarrow F\left[s^{k+1} 0\right]$; thus $\xi \alpha \Vdash F\left[s^{k+1} 0\right]$. Hence the result, by the recurrence hypothesis.

Definition. We denote by $\operatorname{int}(n)$ the formula $\forall x(\forall y(s y \notin x \rightarrow y \notin x), n \notin x \rightarrow 0 \notin x)$.

Theorem 4.15 shows that the formulas $\operatorname{int}(n)$ and $n \varepsilon \widetilde{\mathbb{N}}$ are interchangeable, i.e. the formula $\forall n(\operatorname{int}(n) \leftrightarrow n \varepsilon \widetilde{\mathbb{N}})$ is realized by a proof-like term : this is the storage theorem for integers.

Lemma 4.14. $\lambda g \lambda x(g)(\sigma) x \Vdash \forall y(s y \notin \widetilde{\mathbb{N}} \rightarrow y \notin \widetilde{\mathbb{N}})$.

Proof. We show that $\lambda g \lambda x(g)(\sigma) x \Vdash s b \notin \widetilde{\mathbb{N}} \rightarrow b \notin \widetilde{\mathbb{N}}$ for every individual $b$.

This is obvious if $b$ is not of the form $s^{n} 0$, since then $\|b \notin \widetilde{\mathbb{N}}\|=\emptyset$. Thus, it remains to show :

$\lambda g \lambda x(g)(\sigma) x \Vdash s^{n+1} 0 \notin \widetilde{\mathbb{N}} \rightarrow s^{n} 0 \notin \widetilde{\mathbb{N}}$. Thus, let $\xi \Vdash s^{n+1} 0 \notin \widetilde{\mathbb{N}} ;$ we must show :

$\lambda g \lambda x(g)(\sigma) x \star \xi \cdot \underline{n} \cdot \pi \in \Perp \Perp \Perp$, i.e. $\xi \star \sigma \underline{n} \cdot \pi \in \mathbb{\Perp}$, which is clear, since $\sigma \underline{n}=\underline{n+1}$.

Theorem 4.15 (Storage theorem).

i) $I \Vdash \forall x^{\widetilde{\mathbb{N}}} \operatorname{int}(x)$.

ii) $T \Vdash \forall x(\operatorname{int}(x), x \notin \widetilde{\mathbb{N}} \rightarrow \perp)$ with $T=\lambda n \lambda f((n) \lambda g \lambda x(g)(\sigma) x) f \underline{0}$.

Proof.

i) It is theorem 4.12(i), if we take for $F[x, y]$ the formula $y \notin x$.

ii) Let $\nu \Vdash \operatorname{int}(a), \phi \Vdash a \notin \widetilde{\mathbb{N}}$ and $\pi \in \Pi$. We must show $T \star \nu \cdot \phi \cdot \pi \in \Perp$, that is : $\nu \star \lambda g \lambda x(g)(\sigma) x \cdot \phi \cdot \underline{0} \cdot \pi \in \Perp \Perp$.

By hypothesis, we have $\nu \Vdash \forall y(s y \notin \widetilde{\mathbb{N}} \rightarrow y \notin \widetilde{\mathbb{N}}), a \notin \widetilde{\mathbb{N}} \rightarrow 0 \notin \widetilde{\mathbb{N}}$.

But we have $\underline{0} \cdot \pi \in\|0 \notin \widetilde{\mathbb{N}}\|$ by definition of $\widetilde{\mathbb{N}}$ and, by lemma 4.14: $\lambda g \lambda x(g)(\sigma) x \Vdash \forall y(s y \notin \widetilde{\mathbb{N}} \rightarrow y \notin \widetilde{\mathbb{N}})$. Hence the result.

From theorem 4.12 (ii), it follows immediately that the recurrence scheme of $Z F$ is realized in $\mathcal{N}$; it is the scheme :

$\forall \vec{x}(\forall y(F[\vec{x}, y] \rightarrow F[\vec{x}, s y]), F[\vec{x}, 0] \rightarrow(\forall n \in \widetilde{\mathbb{N}}) F[\vec{x}, n])$ for every formula $F[\vec{x}, y]$ of ZF (i.e. 
written with $\notin, \subseteq, 0, s)$.

Then, indeed, the formula $F$ is compatible with the extensional equivalence $\simeq$.

Since the function $s$ is compatible with $\simeq$, we deduce from lemma 4.14 that the formula: $\forall y(y \in \widetilde{\mathbb{N}} \rightarrow s y \in \widetilde{\mathbb{N}})$ is realized in $\mathcal{N}$; the formula $0 \in \widetilde{\mathbb{N}}$ is also obviously realized.

From the recurrence scheme just proved, we deduce that :

$\widetilde{\mathbb{N}}$ is the set of integers of the model $\mathcal{N}$, considered as a model of $Z F$.

\section{Theorem 4.16.}

i) Let $f: \mathbb{N}^{k} \rightarrow \mathbb{N}$ be a recursive function. Then, the formula : $\forall x_{1}^{\widetilde{\mathbb{N}}} \ldots \forall x_{k}^{\widetilde{\mathbb{N}}}\left(f\left(x_{1}, \ldots, x_{k}\right) \varepsilon \widetilde{\mathbb{N}}\right)$ is realized in $\mathcal{N}$.

ii) Let $g: \mathbb{N}^{k} \rightarrow 2$ be a recursive function. Then, the formula:

$\forall x_{1}^{\widetilde{\mathbb{N}}} \ldots \forall x_{k}^{\widetilde{\mathbb{N}}}\left(g\left(x_{1}, \ldots, x_{k}\right)=1 \vee g\left(x_{1}, \ldots, x_{k}\right)=0\right)$ is realized in $\mathcal{N}$.

i) This can be written $\forall x_{1}^{\text {ent }} \ldots \forall x_{k}^{\text {ent }} \operatorname{ent}\left(f\left(x_{1}, \ldots, x_{k}\right)\right)$. The proof is done in [18, 15].

ii) We have $\mathcal{N} \Vdash\left(\forall x_{1} \varepsilon \mathbf{I N}\right) \ldots\left(\forall x_{k} \varepsilon \mathbb{I N}\right) g\left(x_{1}, \ldots, x_{k}\right) \varepsilon \mathbf{I}$.

Now, since $g$ is recursive, we have, by (i) :

$\mathcal{N} \Vdash\left(\forall x_{1} \varepsilon \widetilde{\mathbb{N}}\right) \ldots\left(\forall x_{k} \varepsilon \widetilde{\mathbb{N}}\right) g\left(x_{1}, \ldots, x_{k}\right) \varepsilon \widetilde{\mathbb{N}}$.

Hence the result, by lemma 4.17 .

Lemma 4.17. $\lambda x \lambda y \lambda f(f) x y \Vdash \forall x^{\beth 2}(x \neq 1, x \neq 0 \rightarrow x \notin \widetilde{\mathbb{N}})$.

Proof. We have to show :

$\lambda x \lambda y \lambda f(f) x y \Vdash \top, \perp \rightarrow 0 \notin \widetilde{\mathbb{N}}$ and $\lambda x \lambda y \lambda f(f) f x y \Vdash \perp, \top \rightarrow 1 \notin \widetilde{\mathbb{N}}$.

Thus let $\xi \Vdash \top$ (i.e. $\xi \in \Lambda$ arbitrary) and $\eta \Vdash \perp$. We have to show :

$\lambda x \lambda y \lambda f(f) x y \star \xi \cdot \eta \cdot \underline{0} \cdot \pi \in \Perp \Perp$ and $\lambda x \lambda y \lambda f(f) x y \star \eta \cdot \xi \cdot \underline{1} \cdot \pi \in \Perp$ which is trivial.

Remarks. i) In the present paper, theorem 4.16 is used only in trivial particular cases.

ii) Let us recall the difference between $\mathbb{I N}$ and $\widetilde{\mathbb{N}}$ (the set of integers in the model $\mathcal{N}$ ) ; we have :

$\xi \Vdash \forall x^{\mathbb{N}} F[x] \quad$ iff $(\forall n \in \mathbb{N})\left(\forall \pi \in\left\|F\left[s^{n} 0\right]\right\|\right) \xi \star \pi \in \Perp$.

$\xi \Vdash \forall x^{\widetilde{\mathbb{N}}} F[x]$ iff $(\forall n \in \mathbb{N})\left(\forall \pi \in\left\|F\left[s^{n} 0\right]\right\|\right) \xi \star \underline{n} \bullet \pi \in \Perp \Perp$.

Notice that we have $K \Vdash \forall x(x \notin \mathbb{N} \rightarrow x \notin \widetilde{\mathbb{N}})$, in other words $K \Vdash \widetilde{\mathbb{N}} \subset \mathbb{I}$. This means that, in $\mathcal{N}$, the set $\widetilde{\mathbb{N}}$ of integers is strongly included in $\mathbb{I N}$. In the particular realizability model considered below (and, in fact, in every non trivial realizability model), the formula $\mathbb{I N} \nsubseteq \widetilde{\mathbb{N}}$ is realized.

\section{Non extensional and dependent choice.}

For each formula $F\left(x, y_{1}, \ldots, y_{m}\right)$ of $\mathrm{ZF}_{\varepsilon}$, we add a function symbol $f_{F}$ of arity $m+1$, with the axiom : $\forall \vec{y}\left(\forall k^{\mathbb{N}} F\left[f_{F}(k, \vec{y}), \vec{y}\right] \rightarrow \forall x F[x, \vec{y}]\right)$

or else : $\forall \vec{y}\left(\forall k^{\text {ent }} F\left[f_{F}(k, \vec{y}), \vec{y}\right] \rightarrow \forall x F[x, \vec{y}]\right)$.

It is the axiom scheme of non extensional choice, in abbreviated form NEAC.

Remarks. i) The axiom scheme NEAC does not imply the axiom of choice in ZF, because we do not suppose that the symbol $f_{F}$ is compatible with the extensional equivalence $\simeq$. It is the reason why we speak about non extensional axiom of choice. On the other hand, as we show below, it implies DC (the axiom of dependent choice).

ii) It seems that we could take for $f_{F}$ a $m$-ary function symbol and use the following simpler (and logically equivalent) axiom scheme NEAC' : $\forall \vec{y}\left(F\left[f_{F}(\vec{y}), \vec{y}\right] \rightarrow \forall x F[x, \vec{y}]\right)$.

But this axiom scheme cannot be realized, even though the axiom scheme NEAC is realized by a 
very simple proof-like term (theorem 4.18), provided the instruction $\varsigma$ is present.

More precisely, we can define a function $f_{F}$ in $\mathcal{M}$, such that NEAC is realized in $\mathcal{N}$, but this is impossible for NEAC'.

Theorem 4.18 (NEAC).

For each closed formula $\forall x \forall \vec{y} F$, we can define a $(m+1)$-ary function symbol $f_{F}$ such that : $\lambda x(\varsigma) x x \Vdash \forall \vec{y}\left(\forall k^{e n t} F\left[f_{F}(k, \vec{y}) / x, \vec{y}\right] \rightarrow \forall x F[x, \vec{y}]\right)$.

Proof. For each $k \in \mathbb{N}$ we put $P_{k}=\left\{\pi \in \Pi ; \xi \star \underline{k} \cdot \pi \notin \Perp, k=\mathrm{n}_{\xi}\right\}$.

For each individual $x$, we have : $\|\forall x F[x, \vec{y}]\|=\bigcup_{a}\|F[a, \vec{y}]\|$.

Therefore, there exists a function $f_{F}$ such that, given $k \in \mathbb{N}$ and $\vec{y}$ such that $P_{k} \cap\|\forall x F[x, \vec{y}]\| \neq \emptyset$, we have $P_{k} \cap\left\|F\left[f_{F}(k, \vec{y}), \vec{y}\right]\right\| \neq \emptyset$.

Now, we want to show $\lambda x(\varsigma) x x \Vdash \forall k^{\text {ent }} F\left[f_{F}(k, \vec{y}), \vec{y}\right] \rightarrow F[x, \vec{y}]$, for every individuals $x, \vec{y}$. Thus, let $\xi \Vdash \forall k^{\mathrm{ent}} F\left[f_{F}(k, \vec{y}), \vec{y}\right]$ and $\pi \in\|F[a, \vec{y}]\|$; we must show $\lambda x(\varsigma) x x \star \xi \bullet \pi \in \Perp$. If this is false, we have $\varsigma \star \xi \cdot \xi \cdot \pi \notin \Perp$ and therefore $\xi \star \underline{j} \cdot \pi \notin \Perp$ with $j=\mathrm{n}_{\xi}$. It follows that $\pi \in P_{j} \cap\|F[a, \vec{y}]\|$; thus, there exists $\pi^{\prime} \in P_{j} \cap\left\|F\left[f_{F}(j, \vec{y}), \vec{y}\right]\right\|$. Now, we have $j \cdot \pi^{\prime} \in\left\|\forall k^{\text {ent }} F\left[f_{F}(k, \vec{y}), \vec{y}\right]\right\|$, and therefore, by hypothesis on $\xi$, we have : $\xi \star \underline{j} \cdot \pi^{\prime} \in \Perp \Perp$. This is in contradiction with $\pi^{\prime} \in P_{j}$.

NEAC implies DC. Let us call DCS (dependent choice scheme) the following axiom scheme : $\forall \vec{z}\left(\forall x \exists y F[x, y, \vec{z}] \rightarrow \forall n^{\mathrm{ent}} \exists ! y S_{F}[n, y, \vec{z}] \wedge \forall n^{\mathrm{ent}} \exists y \exists y^{\prime}\left\{S_{F}[n, y, \vec{z}], S_{F}\left[s n, y^{\prime}, \vec{z}\right], F\left[y, y^{\prime}, \vec{z}\right]\right\}\right)$. where $F$ is a formula of $\mathrm{ZF}_{\varepsilon}$ with free variables $x, y, \vec{z}$; the formula $S_{F}$ is written below. In the following, we omit the variables $\vec{z}$ (the parameters), for sake of simplicity. The usual axiom of dependent choice DC is obtained by taking for $F\left[x, y, z_{0}, z_{1}\right]$ the formula $y \varepsilon z_{0} \wedge\left(x \varepsilon z_{0} \rightarrow<x, y>\varepsilon z_{1}\right)$.

We now show how to define the formula $S_{F}$, so that $\mathrm{ZF}_{\varepsilon}$, NEAC $\vdash \mathrm{DCS}$; we shall conclude that $\mathrm{DC}$ is realized.

So, let us assume $\forall x \exists y F[x, y]$. By NEAC, there is a function symbol $f$ such that: $\forall x \exists k^{\text {ent }} F[x, f(k, x)]$. We define the formula $R_{F}[x, y]$ as follows :

$R_{F}[x, y] \equiv \exists k^{\text {ent }}\left\{F[x, f(k, x)], \forall i^{\text {ent }}(i<k \rightarrow \neg F[x, f(i, x)]), y=f(k, x)\right\}$. This means : " $y=f(k, x)$ for the first integer $k$ such that $F[x, f(k, x)]$ ".

Therefore, $R_{F}$ is functional, i.e. we have $\forall x \exists ! y R_{F}(x, y)$.

$S_{F}$ is defined so as to represent a sequence obtained by iteration of the function given by $R_{F}$, beginning (arbitrarily) at 0 :

$S_{F}(n, x) \equiv \forall z\left[\forall m \forall y \forall y^{\prime}\left(<m, y>\varepsilon z, R_{F}\left(y, y^{\prime}\right) \rightarrow<s m, y^{\prime}>\varepsilon z\right),<0,0>\varepsilon z \rightarrow<n, x>\varepsilon z\right]$.

It should be clear that, with this definition of $S_{F}$, we obtain :

$\forall n^{\mathrm{ent}} \exists ! y S_{F}[n, y]$ and $\forall n^{\mathrm{ent}} \exists y \exists y^{\prime}\left\{S_{F}[n, y], S_{F}\left[s n, y^{\prime}\right], F\left[y, y^{\prime}\right]\right\}$.

Thus, DCS is provable from $\mathrm{ZF}_{\varepsilon}$ and NEAC.

Remark. We have used the binary function symbol $\langle x, y\rangle$ which is defined, in the ground model $\mathcal{M}$, in the usual way : $\langle a, b\rangle=\{\{a\},\{a, b\}\}$. Then, the formulas:

$\forall x \forall x^{\prime} \forall y \forall y^{\prime}\left(<x, y>=<x^{\prime}, y^{\prime}>\hookrightarrow x=x^{\prime}\right), \quad \forall x \forall x^{\prime} \forall y \forall y^{\prime}\left(<x, y>=<x^{\prime}, y^{\prime}>\hookrightarrow y=y^{\prime}\right)$, are trivially realized by $I$. 


\section{Properties of the Boolean algebra $\mathrm{I2}$.}

Let $(x<y)$ be the binary recursive function defined as follows in $\mathcal{M}$ :

$(m<n)=1$ if $m, n \in \mathbb{N}, m<n$; else $(m<n)=0$.

Theorem 4.19. For every choice of $\Perp$, the relation $(x<y)=1$ is, in $\mathcal{N}$, a strict well founded partial order, which is the usual order on integers (i.e. on $\widetilde{\mathbb{N}}$ ).

Proof. Indeed, the formulas :

$\forall x((x<x) \neq 1)$ and $\forall x \forall y \forall z((x<y)=1 \hookrightarrow((y<z)=1 \hookrightarrow(x<z)=1))$

are trivially realized.

Moreover, since the relation $(x<y)=1$ is well founded, we have (theorem 4.9) :

$\mathrm{Y} \Vdash \forall x(\forall y((y<x)=1 \hookrightarrow F[y]) \rightarrow F[x]) \rightarrow \forall x F[x]$

for every formula $F[x]$ with parameters and one free variable.

By theorem 4.16(ii), the binary recursive function $(x<y)$ sends $\widetilde{\mathbb{N}}^{2}$ into $\{0,1\}$, in the model $\mathcal{N}$. Therefore, it suffices to check that the following formulas are realized in $\mathcal{N}$ :

$\forall x^{\widetilde{\mathbb{N}}} \forall y^{\widetilde{\mathbb{N}}}(y \leq x \rightarrow(x<y) \neq 1) ; \forall x^{\widetilde{\mathbb{N}}} \forall y^{\widetilde{\mathbb{N}}}(x<y \rightarrow(x<y)=1)$.

Now the following formulas are trivially realized :

$\forall x^{\mathbb{I N}} \forall y^{\mathbb{I N}} \forall z^{\mathbb{I N}}(x=y+z \rightarrow(x<y) \neq 1) ; \forall x^{\mathbb{I N}} \forall y^{\mathbb{I N}} \forall z^{\mathbb{I N}}(y=x+z+1 \rightarrow(x<y)=1)$.

In the ground model $\mathcal{M}$, we put, for each integer $n$ :

$$
\mathbf{n}=\{0,1, \ldots, n-1\}=\left\{0, s 0, \ldots, s^{n-1} 0\right\} .
$$

The functions $n \longmapsto \mathbf{n}$ and $n \longmapsto$ In are defined in the realizability model $\mathcal{N}$, with domain $\mathfrak{I N}$.

\section{Theorem 4.20.}

The following formulas are realized in $\mathcal{N}$ :

i) $\forall x^{\mathbb{I N}} \forall m^{\mathbb{I N}}((x<m)=1 \leftrightarrow x \varepsilon \mathbf{I m})$;

ii) $\forall m^{\mathbb{I N}} \forall n^{\mathbb{I N}}((m<n)=1 \rightarrow \mathbf{I m} \subset \mathbf{I n})$;

iii) $\forall x^{\mathbb{I N}} \forall m^{\mathbb{I N}}\left((x<m)=1 \leftrightarrow \exists y^{\mathbb{I N}}(m=x+y+1)\right)$.

Proof. Remember that $x \subset y$ is the formula $\forall z(z \notin y \rightarrow z \notin x)$.

i) We have trivially $\|(a<m) \neq 1\|=\|a \notin \mathbf{I m}\|$ for every $a, m \in \mathbb{N}$.

ii) By transitivity of the relation $(m<n)=1$ (theorem 4.19).

iii) We observe that $\|(a<m) \neq 1\|=\|(\forall y \varepsilon \mathbb{I N})(m \neq a+y+1)\|$ for every $a, m \in \mathbb{N}$.

For each $n \varepsilon \mathbb{I N}$ (and, in particular, for each $n \varepsilon \widetilde{\mathbb{N}}$, i.e. for each integer of $\mathcal{N}$ ), the set defined, in $\mathcal{N}$, by $(x<n)=1$ (the strict initial segment defined by $n$ ) is therefore extensionally equivalent to In.

Theorem 4.21. In $\mathcal{N}$, the application $(x, y) \longmapsto m y+x$ is a bijection from $\mathbf{I m} \times \mathbf{I n}$ onto $I(\mathbf{m n})$. Indeed, the following formulas are realized in $\mathcal{N}$ by $I$ :

i) $\forall m^{\mathbb{I N}} \forall n^{\mathbb{I N}} \forall x^{\mathbf{I m}} \forall y^{\mathrm{In}}((m y+x) \varepsilon \mathbf{I m n})$;

ii) $\forall m^{\beth \mathbb{N}} \forall n^{\beth \mathbb{N}} \forall x^{\beth \mathbf{m}} \forall x^{\prime \mathbf{I} \mathbf{m}} \forall y^{\mathcal{I n}} \forall y^{\prime \mathbf{I n}}\left(m y+x=m y^{\prime}+x^{\prime} \hookrightarrow x=x^{\prime}\right)$; $\forall m^{\beth \mathbb{N}} \forall n^{\beth \mathbb{N}} \forall x^{\beth \mathbf{m}} \forall x^{\prime \mathbf{I m}} \forall y^{\beth \mathbf{n}} \forall y^{\prime \mathbf{I n}}\left(m y+x=m y^{\prime}+x^{\prime} \hookrightarrow y=y^{\prime}\right) ;$

iii) $\forall m^{\beth \mathbb{N}} \forall n^{\mathbb{I N}} \forall z^{\beth \mathbf{m n}} \exists x^{\beth \mathbf{m}} \exists y^{\beth \mathbf{n}}(z=m y+x)$.

Proof.

i) and ii) We simply have to replace $\forall m^{\beth \mathbb{N}}$ and $\forall x^{\beth m}$ with their definitions, which are : $\forall m^{\mathbb{I N}} F \equiv \forall m\left(1_{\mathbb{N}}(m)=1 \hookrightarrow F\right) ; \forall x^{\beth \mathbf{m}} F \equiv \forall x((x<m)=1 \hookrightarrow F)$.

We see immediately that these two formulas are realized by $I$. 
iii) We show that:

$I \Vdash \forall m^{\mathbb{I N}} \forall n^{\mathbb{I N}} \forall z^{\mathbb{I N}}\left(\forall x^{\mathbb{I N}} \forall y^{\mathbb{I N}}((x<m)=1 \hookrightarrow((y<n)=1 \hookrightarrow z \neq m y+x)) \rightarrow(z<m n) \neq 1\right)$.

Thus, we consider :

$m, n, z_{0} \in \mathbb{N} ; \quad \xi \in \Lambda, \xi \Vdash \forall x^{\mathbb{I N}} \forall y^{\mathbb{N}}((x<m)=1 \hookrightarrow((y<n)=1 \hookrightarrow z \neq m y+x))$

and $\pi \in\left\|\left(z_{0}<m n\right) \neq 1\right\|$. We must show $I \star \xi \bullet \pi \in \Perp \Perp \Perp$, that is $\xi \star \pi \in \Perp \Perp$.

We have $\left\|\left(z_{0}<m n\right) \neq 1\right\| \neq \emptyset$, therefore $z_{0}<m n$.

Thus, there exist $x_{0}, y_{0} \in \mathbb{N}, x_{0}<m, y_{0}<n$ such that $z_{0}=m x_{0}+y_{0}$.

Now, by hypothesis on $\xi$, we have :

$\xi \Vdash\left(x_{0}<m\right)=1 \hookrightarrow\left(\left(y_{0}<n\right)=1 \hookrightarrow z_{0} \neq m y_{0}+x_{0}\right)$, in other words $\xi \Vdash \perp$.

Injection of In into $\mathcal{P}(\widetilde{\mathbb{N}})$. Remember that we have fixed a recursive bijection $: \xi \longmapsto \mathrm{n}_{\xi}$ from $\Lambda$ onto $\mathbb{N}$. The inverse bijection will be denoted $n \longmapsto \xi_{n}$.

This bijection is used in the execution rule of the instruction $\varsigma$, which is as follows :

$$
\varsigma \star \xi \cdot \eta \cdot \pi \succ \xi \star \underline{\mathrm{n}}_{\eta} \cdot \pi \text {. }
$$

We define, in $\mathcal{M}$, a function $\Delta: \mathbb{N} \rightarrow 2$ by putting $\Delta(n)=0 \Leftrightarrow \xi_{n} \Vdash \perp$.

In this way, we have defined a function symbol $\Delta$, in the language of $\mathrm{ZF}_{\varepsilon}$. In the realizability model $\mathcal{N}$, the symbol $\Delta$ represents a function from $\mathbb{I N}$ into $\mathbf{I 2}$. In particular, the function $\Delta$ sends the set $\widetilde{\mathbb{N}}$ of integers of the model $\mathcal{N}$ into the Boolean algebra $\mathbf{I 2}$.

Theorem 4.22. Let us put $\theta=\lambda x \lambda y(\varsigma) y x x$; then, we have :

$$
\theta \Vdash \forall x^{\beth 2}\left(x \neq 0 \rightarrow \exists n^{e n t}\{\Delta(n) \neq 0, \Delta(n) \leq x\}\right)
$$

where $\leq$ is the order relation of the Boolean algebra $\mathbf{I 2}: y \leq x$ is the formula $x=(y \vee x)$.

Proof. We must show $\theta \Vdash \forall x^{\beth 2}\left(x \neq 0, \forall n^{\text {ent }}(\Delta(n) \neq 0 \rightarrow x \neq \Delta(n) \vee x) \rightarrow \perp\right)$.

Thus, let $a \in\{0,1\}, \quad \xi \Vdash a \neq 0, \quad \eta \Vdash \forall n^{\text {ent }}(\Delta(n) \neq 0 \rightarrow a \neq \Delta(n) \vee a)$ and $\pi \in \Pi$.

We must show $\theta \star \xi \cdot \eta \cdot \pi \in \Perp \Perp$ that is $\varsigma \star \eta \cdot \xi \cdot \xi \cdot \pi \in \Perp \Perp \Perp$, or else $\eta \star \underline{\mathrm{n}}_{\xi} \cdot \xi \cdot \pi \in \Perp \Perp$.

By hypothesis on $\eta$, it suffices to show $\underline{\mathrm{n}}_{\xi} \cdot \xi \cdot \pi \in\left\|\forall n^{\mathrm{ent}}(\Delta(n) \neq 0 \rightarrow a \neq \Delta(n) \vee a)\right\|$, that is, by definition of the quantifier $\forall n^{\text {ent }}: \quad \xi \cdot \pi \in\left\|\Delta\left(\mathrm{n}_{\xi}\right) \neq 0 \rightarrow a \neq \Delta\left(\mathrm{n}_{\xi}\right) \vee a\right\|$.

This amounts to show $\xi \Vdash \Delta\left(\mathrm{n}_{\xi}\right) \neq 0$ and $a=\Delta\left(\mathrm{n}_{\xi}\right) \vee a$.

- Proof of $\xi \| \Delta\left(\mathrm{n}_{\xi}\right) \neq 0$ : if $\Delta\left(\mathrm{n}_{\xi}\right)=1$, this is trivial, because $\left\|\Delta\left(\mathrm{n}_{\xi}\right) \neq 0\right\|=\emptyset$; if $\Delta\left(\mathrm{n}_{\xi}\right)=0$, then $\xi \Vdash \perp$, by definition of $\Delta$.

- Proof of $a=\Delta\left(\mathrm{n}_{\xi}\right) \vee a$ : this is obvious if $a=1$; if $a=0$, then $\xi \Vdash \perp$, by hypothesis on $\xi$. Therefore $\Delta\left(\mathrm{n}_{\xi}\right)=0$ by definition of $\Delta$, hence the result.

By theorem 4.22 , the set $\{\Delta(n) ; n \varepsilon \widetilde{\mathbb{N}}, \Delta(n) \neq 0\}$ is, in the realizability model $\mathcal{N}$, a countable dense subset of the Boolean algebra $\mathbf{I 2}$ : this means that each element $\neq 0$ of this Boolean algebra has a lower bound of the form $\Delta(n)$, with $n \varepsilon \widetilde{\mathbb{N}}$ and $\Delta(n) \neq 0$.

It follows that the application of $\mathbf{I 2}$ into $\mathcal{P}(\widetilde{\mathbb{N}})$ given by :

$$
x \longmapsto\{n \varepsilon \widetilde{\mathbb{N}} ; \Delta(n) \leq x, \Delta(n) \neq 0\}
$$

is one to one : indeed, if $a, b \varepsilon \beth 2$ with $a \neq b$, then $a+b \neq 0$; thus, there exists an integer $n \varepsilon \widetilde{\mathbb{N}}$ such that $\Delta(n) \neq 0$ and $\Delta(n) \leq a+b$. Therefore, we have $\Delta(n) \leq a$ iff $(b \wedge \Delta(n))=0$.

But, since $\Delta(n) \neq 0$, we get : $\Delta(n) \leq a$ iff $\Delta(n) \not \leq b$.

We have shown :

Theorem 4.23.

The formula : "there exists an injection of $\mathbf{\beth} 2$ into $\mathcal{P}(\widetilde{\mathbb{N}})$ " is realized in the model $\mathcal{N}$. 
Corollary 4.24. The formula : "for every integer $n$ there exists an injection of In into $\mathcal{P}(\widetilde{\mathbb{N}})$ " is realized in the model $\mathcal{N}$.

Proof. Using theorem 4.21 we see, by recurrence on $m$, that the model $\mathcal{N}$ realizes the formula :

" $\forall m^{\widetilde{N}}\left((\mathbf{I} \mathbf{2})^{m}\right.$ is equipotent to $\left.\mathbf{I}\left(\mathbf{2}^{\mathbf{m}}\right)\right) " ;$ and therefore also the formula :

" $\forall m^{\widetilde{\mathbb{N}}}$ (there exists an injection of $\mathbf{I}\left(\mathbf{2}^{\mathbf{m}}\right)$ into $\left.\mathcal{P}(\widetilde{\mathbb{N}})\right)$ ".

Finally, by theorem 4.20(ii), we see that the following formula is realized :

" $\forall n^{\widetilde{\mathbb{N}}}$ (there exists an injection of In into $\left.\mathcal{P}(\widetilde{\mathbb{N}})\right)$ ".

\section{REALIZABILITy MODELS IN Which $\mathbb{R}$ IS NOT WELL ORDERED}

\section{I2 atomless.}

Theorem 5.1. We suppose there exist two proof-like terms $\omega_{0}, \omega_{1}$ such that, for every $\pi \in \Pi$, we have $\omega_{0} k_{\pi} \Vdash \perp$ or $\omega_{1} k_{\pi} \Vdash \perp$. Then, the Boolean algebra $\mathbf{I}$ is non trivial. Indeed : $\theta \Vdash \forall x(x \neq 1, x \neq 0 \rightarrow x \notin \mathbf{I} 2) \rightarrow \perp$ with $\theta=\lambda f(\mathbf{c c}) \lambda k\left((f)\left(\omega_{1}\right) k\right)\left(\omega_{0}\right) k$.

Proof. Let $\xi \Vdash \forall x(x \neq 1, x \neq 0 \rightarrow x \notin \beth 2)$ and $\pi \in \Pi$. We must show :

$\theta \star \xi \cdot \pi \in \Perp$, that is $\xi \star \omega_{1} \mathrm{k}_{\pi} \cdot \omega_{0} \mathrm{k}_{\pi} \cdot \pi \in \Perp$.

But, by hypothesis on $\xi$, we have $\xi \Vdash \top, \perp \rightarrow \perp$ and $\xi \Vdash \perp, \top \rightarrow \perp$. Hence the result, by hypothesis on $\omega_{1}, \omega_{0}$.

Remark. When the Boolean algebra $\mathbf{\beth 2}$ is non trivial, there are necessarily non standard integers in the realizability model $\mathcal{N}$, i.e. integers which are not in $\mathcal{M}$. Indeed, let $a \varepsilon \mathbf{I 2}, a \neq 0,1$; by theorem 4.22, there is an integer $n$ such that $\Delta(n) \neq 0, \Delta(n) \leq a$; thus $\Delta(n) \neq 1$. The integer $n$ cannot be standard, since $\Delta(m)=0$ or 1 if $m$ is in $\mathcal{M}$.

Theorem 5.2. We suppose that there exists three proof-like terms $\alpha_{0}, \alpha_{1}, \alpha_{2}$ such that, for every $\xi \in \Lambda$ and $\pi \in \Pi$, we have $k_{\pi} \xi \alpha_{0} \Vdash \perp$ or $k_{\pi} \xi \alpha_{1} \Vdash \perp$ or $k_{\pi} \xi \alpha_{2} \Vdash \perp$.

Then, the Boolean algebra $\mathbf{I} \mathbf{2}$ is atomless. Indeed:

$\theta \Vdash \forall x[\forall y(x \wedge y \neq 0, x \wedge y \neq x \rightarrow y \notin \beth 2), x \neq 0 \rightarrow x \notin \mathbf{I 2}]$

with $\theta=\lambda x \lambda y(\mathbf{c c}) \lambda k\left((x)(k) y \alpha_{0}\right)\left((x)(k) y \alpha_{1}\right)(k) y \alpha_{2}$.

Proof. By a simple computation, we see that we must show :

i) $\theta \Vdash(\perp, \perp \rightarrow \perp), \perp \rightarrow \perp$.

ii) $\theta \||\top, \perp \rightarrow \perp| \cap|\perp, \top \rightarrow \perp|, \top \rightarrow \perp$.

Proof of (i) : let $\eta \in|\perp, \perp \rightarrow \perp|$ and $\xi \in|\perp|$. We must show $\theta \star \eta \cdot \xi \cdot \pi \in \Perp$, that is :

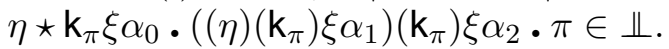

But, from $\xi \Vdash \perp$, we deduce $\mathrm{k}_{\pi} \xi \zeta \Vdash \perp$ for every $\zeta \in \Lambda_{c}$.

Since $\eta \Vdash \perp, \perp \rightarrow \perp$, we have $\left((\eta)\left(\mathbf{k}_{\pi}\right) \xi \alpha_{1}\right)\left(\mathbf{k}_{\pi}\right) \xi \alpha_{2} \Vdash \perp$ and therefore :

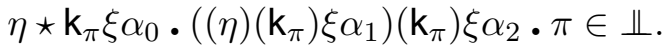

Proof of (ii) : let $\eta \in|\top, \perp \rightarrow \perp| \cap|\perp, \top \rightarrow \perp|$ and $\xi \in \Lambda_{c}$. Again, we must show that :

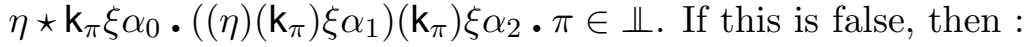

$\mathrm{k}_{\pi} \xi \alpha_{0} \nVdash \perp$ (because $\eta \Vdash \perp, \top \rightarrow \perp$ ) and

$\left((\eta)\left(\mathrm{k}_{\pi}\right) \xi \alpha_{1}\right)\left(\mathrm{k}_{\pi}\right) \xi \alpha_{2} \nVdash \leftarrow \perp$ (because $\eta \Vdash \top, \perp \rightarrow \perp$ ).

But, since $\eta \Vdash \perp, \top \rightarrow \perp$ (resp. $\top, \perp \rightarrow \perp$ ), we have $\mathbf{k}_{\pi} \xi \alpha_{1} \nVdash \perp$ (resp. $\left.\mathbf{k}_{\pi} \xi \alpha_{2} \nVdash \perp\right)$.

This contradicts the hypothesis of the theorem. 


\section{$\mathbb{R}$ not well orderable.}

\section{Theorem 5.3.}

We suppose that there exists a proof-like term $\omega$ such that, for every $\xi, \xi^{\prime} \in \Lambda, \xi \neq \xi^{\prime}$ and $\pi \in \Pi$, we have $\omega k_{\pi} \xi \Vdash \perp$ or $\omega k_{\pi} \xi^{\prime} \Vdash \perp$.

Then we have, for every formula $F$ with three free variables : $\theta \Vdash \forall m^{\mathbb{I N}} \forall n^{\mathbb{I N}} \forall z[(m<n)=1 \hookrightarrow$

$$
\left.\left(\forall x \forall y \forall y^{\prime}\left(F(x, y, z), F\left(x, y^{\prime}, z\right), y \neq y^{\prime} \rightarrow \perp\right), \forall y^{\beth \mathbf{n}_{\neg}} \neg x^{\beth \mathbf{m}_{\neg}} F(x, y, z) \rightarrow \perp\right)\right]
$$

with $\theta=\lambda x \lambda x^{\prime}(\mathrm{cc}) \lambda k\left(x^{\prime}\right) \lambda z(x z z)(\omega) k z$.

Remark. This shows that, if $(m<n)=1$, then $(\mathbf{I m} \subset \mathbf{I n}$ and) there is no surjection of $\mathbf{I m}$ onto In : indeed, it suffices to take, for $F(x, y, z)$, the formula $\langle x, y\rangle \varepsilon z$.

Proof. Assume this is false ; then, there exist $m, n \in \mathbb{N}$ with $m<n$, an individual $c$, two terms $\xi, \xi^{\prime} \in \Lambda$ and a stack $\pi \in \Pi$ such that :

$\theta \star \xi \cdot \xi^{\prime} \cdot \pi \notin \Perp ;$

$\xi \Vdash \forall x \forall y \forall y^{\prime}\left[F(x, y, c), F\left(x, y^{\prime}, c\right), y \neq y^{\prime} \rightarrow \perp\right]$;

$\xi^{\prime} \Vdash \forall y^{\beth \mathbf{n}} \neg \forall x^{I \mathbf{m}} \neg F(x, y, c)$.

Therefore, we have $\xi^{\prime} \star \eta \cdot \pi \notin \Perp$ with $\eta=\lambda z(\xi z z)(\omega) \mathrm{k}_{\pi} z$. By hypothesis on $\xi^{\prime}$ we have, for every integer $i<n: \eta \nVdash \forall x^{\beth \mathbf{m}_{\neg F}}(x, i, c)$. Thus, there exists an integer $m_{i}<m$ such that $\eta \nVdash \neg F\left(m_{i}, i, c\right)$. It follows that there exist $\xi_{i} \in \Lambda$ and $\pi_{i} \in \Pi$ such that $\xi_{i} \Vdash F\left(m_{i}, i, c\right)$ and $\eta \star \xi_{i} \cdot \pi_{i} \notin \Perp \Perp$. By definition of $\eta$, we get $\xi \star \xi_{i} \cdot \xi_{i} \cdot \omega \mathrm{k}_{\pi} \xi_{i} \cdot \pi_{i} \notin \Perp \Perp$. By hypothesis on $\xi$, it follows that $\omega \mathrm{k}_{\pi} \xi_{i} \|<i \neq i$; in other words, we have $\omega \mathrm{k}_{\pi} \xi_{i} \| \leftarrow \perp$ for every integer $i<n$.

By the hypothesis of the theorem, it follows that we have $\xi_{i}=\xi_{j}$ for every $i, j<n$.

But, since $m_{i}<m<n$ and $i<n$, there exist $i, j<n, i \neq j$ such that $m_{i}=m_{j}=k$. Then, $\xi_{i}=\xi_{j} \Vdash F(k, i, c), F(k, j, c)$ and $\omega \mathrm{k}_{\pi} \xi_{i} \Vdash i \neq j$ since $\|i \neq j\|=\emptyset$.

Therefore, by hypothesis on $\xi$, we have $\xi \star \xi_{i} \bullet \xi_{i} \bullet \omega \mathbf{k}_{\pi} \xi_{i} \bullet \pi_{i} \in \Perp \Perp \Perp$, which is a contradiction.

Now, we see that, with the hypothesis of theorem [5.3, there is no surjection from $\mathbf{I 2}$ onto $\mathbf{I} \times \mathbf{I 2}$. Indeed, by theorem 4.21, there exists a bijection from $\mathbf{I 2} \times \mathbf{I 2}$ onto $\mathbf{I 4}$ and, by theorem [5.3, there is no surjection from $\mathbf{I 2}$ onto $\mathbf{I 4}$. But, by theorem [5.2, $\mathbf{I 2}$ is infinite; it follows that $\mathbf{I 2}$ cannot be well ordered.

Now, by theorem 4.23, $\mathbf{I 2}$ is equipotent with a subset of $\mathcal{P}(\widetilde{\mathbb{N}})$. Therefore, the hypothesis of theorems 5.2 and 5.3 are sufficient in order that the following formula be realized in the model $\mathcal{N}$ :

There is no well ordering on the set of reals.

In fact, the hypothesis of theorem 5.3 is sufficient : this follows from theorem 5.4 ,

Theorem 5.4. Same hypothesis as theorem 5.3: there exists a proof-like term $\omega$ such that, for every $\pi \in \Pi$ and $\xi, \xi^{\prime} \in \Lambda, \xi \neq \xi^{\prime}$, we have $\omega k_{\pi} \xi \Vdash \perp$ or $\omega k_{\pi} \xi^{\prime} \Vdash \perp$.

Then we have, for every formula $F$ with three free variables :

$\theta \Vdash \forall z\left\{\forall x\left[\forall n^{e n t} F(n, x, z) \rightarrow x \notin \mathbf{I 2}\right], \forall n \forall x \forall y[\neg F(n, x, z) \neg F(n, y, z), x \neq y \rightarrow \perp] \rightarrow \perp\right\}$

with $\theta=\lambda x \lambda x^{\prime}(\mathrm{cc}) \lambda k(x) \lambda n(\mathrm{cc}) \lambda h\left(x^{\prime} h h\right)(\omega k) \lambda f(f) h n$.

Remark. This formula means that, in the realizability model $\mathcal{N}$, there is no surjection from the set of integers $\widetilde{\mathbb{N}}$ onto $\mathbf{I 2}$ : it suffices to take for $F(x, y, z)$ the formula $\langle x, y\rangle \notin z$ (the graph of an hypothetical surjection being $\langle x, y\rangle \varepsilon z)$. 
Proof. Reasoning by contradiction, we suppose that there is an individual $c$, a stack $\pi \in \Pi$, and two terms $\xi, \xi^{\prime}$ such that :

$\left.\xi \Vdash \forall x\left[\forall n^{\text {ent }} F(n, x, c) \rightarrow x \notin\right] 2\right] ; \quad \xi^{\prime} \Vdash \forall n \forall x \forall y[\neg F(n, x, c) \neg F(n, y, c), x \neq y \rightarrow \perp]$ and $\theta \star \xi \cdot \xi^{\prime} \cdot \pi \notin \Perp$.

Therefore, we have $\xi \star \eta \cdot \pi \notin \Perp$, with $\eta=\lambda n(\mathrm{cc}) \lambda h\left(\xi^{\prime} h h\right)\left(\omega \mathrm{k}_{\pi}\right) \lambda f(f) h n$.

By hypothesis on $\xi$, we have $\eta \nVdash \forall \forall n^{\text {ent }} F(n, 0, c)$ and $\eta \nVdash \forall n^{\text {ent }} F(n, 1, c)$. Thus, we see that there exist $n_{0}, n_{1} \in \mathbb{N}, \pi_{0} \in\left\|F\left(n_{0}, 0, c\right)\right\|$ and $\pi_{1} \in\left\|F\left(n_{1}, 1, c\right)\right\|$ such that $\eta \star \underline{n}_{0} \bullet \pi_{0} \notin \Perp$ and $\eta \star \underline{n}_{1} \cdot \pi_{1} \notin \Perp$. By performing these two processes, we obtain :

$\xi^{\prime} \star \mathrm{k}_{\pi_{0}} \cdot \mathrm{k}_{\pi_{0}} \cdot \zeta_{0} \cdot \pi_{0} \notin \Perp$ et $\xi^{\prime} \star \mathrm{k}_{\pi_{1}} \cdot \mathrm{k}_{\pi_{1}} \cdot \zeta_{1} \cdot \pi_{1} \notin \Perp \Perp$,

with $\zeta_{0}=\left(\omega \mathbf{k}_{\pi}\right) \lambda f(f) \mathbf{k}_{\pi_{0}} \underline{n}_{0}$ and $\zeta_{1}=\left(\omega \mathbf{k}_{\pi}\right) \lambda f(f) \mathbf{k}_{\pi_{1}} \underline{n}_{1}$.

By hypothesis on $\xi^{\prime}$, we have $\xi^{\prime} \Vdash \neg F\left(n_{0}, 0, c\right), \neg F\left(n_{0}, 0, c\right), 0 \neq 0 \rightarrow \perp$.

Since $\mathrm{k}_{\pi_{0}} \Vdash \neg F\left(n_{0}, 0, c\right)$, we see that $\zeta_{0} \nVdash \perp$ and, in the same way, $\zeta_{1} \nVdash \perp$.

Thus, by the hypothesis of the theorem, we have :

$\lambda f(f) \mathbf{k}_{\pi_{0}} \underline{n}_{0}=\lambda f(f) \mathbf{k}_{\pi_{1}} \underline{n}_{1}$, and therefore $n_{0}=n_{1}$ and $\pi_{0}=\pi_{1}$.

But, we have $\xi^{\prime} \Vdash \neg F\left(n_{0}, 0, c\right), \neg F\left(n_{0}, 1, c\right), 0 \neq 1 \rightarrow \perp$. Moreover, we have :

$\pi_{0} \in\left\|F\left(n_{0}, 0, c\right)\right\|$ and $\pi_{1} \in\left\|F\left(n_{1}, 1, c\right)\right\|$, thus $\pi_{0} \in\left\|F\left(n_{0}, 1, c\right)\right\|$ since $n_{0}=n_{1}, \pi_{0}=\pi_{1}$.

Therefore $\mathrm{k}_{\pi_{0}} \Vdash \neg F\left(n_{0}, 0, c\right)$ and $\neg F\left(n_{0}, 1, c\right)$. Moreover, we have obviously $\zeta_{0} \Vdash 0 \neq 1$, since $\|0 \neq 1\|=\emptyset$. Therefore, we have $\xi^{\prime} \star \mathrm{k}_{\pi_{0}} \cdot \mathrm{k}_{\pi_{0}} \cdot \zeta_{0} \cdot \pi_{0} \in \Perp \Perp \Perp$, which is a contradiction.

Theorems 5.3 and 5.4 show that $\mathbf{I 2}$ is infinite and not equipotent with $\mathbf{I 2} \times \mathbf{I 2}$, thus not well orderable. Since $\mathbf{I 2}$ is equipotent with a subset of $\mathcal{P}(\widetilde{\mathbb{N}})$ (theorem 4.23), we have shown that $\mathcal{P}(\widetilde{\mathbb{N}})$ is not well orderable, with the hypothesis of theorem 5.3 .

More precisely, by corollary 4.24, we know that In is equipotent with a subset of $\mathcal{P}(\widetilde{\mathbb{N}})$ for each integer $n$. Therefore, we have :

Theorem 5.5. With the hypothesis of theorem 5.3, the following formula is realized:

" There exists a sequence $\mathcal{X}_{n}$ of infinite subsets of $\mathcal{P}(\widetilde{\mathbb{N}})$ such that, for every integers $m, n \geq 2$ :

- there is an injection from $\mathcal{X}_{n}$ into $\mathcal{X}_{n+1}$;

- there is no surjection from $\mathcal{X}_{n}$ onto $\mathcal{X}_{n+1}$;

- $\mathcal{X}_{m} \times \mathcal{X}_{n}$ and $\mathcal{X}_{m n}$ are equipotent ".

For each integer $n \geq 2$, the set $\mathbf{n}=\{0,1, \ldots, n-1\}$ is a ring : the ring of integers modulo $n$; the Boolean algebra $\{0,1\}$ is a set of idempotents in this ring. These ring operations extend to the realizability model, giving a ring structure on $\mathbf{I n}$, and $\mathbf{I 2}$ is a set of idempotents in In.

For each $a \varepsilon \mathbf{I 2}$, the equation $a x=x$ defines an ideal in $\mathbf{I n}$, which we denote as $a \mathbf{I n}$.

The application $x \longmapsto a x$ is a retraction from In onto $a \mathbf{I n}$.

Proposition 5.6. The following formulas are realized in $\mathcal{N}$ :

i) $\forall n^{\mathbb{I N}} \forall a^{\mathrm{I2}}$ (the application $x \longmapsto(a x,(1-a) x)$ is a bijection

ii) $\forall m^{\mathbb{I N}} \forall n^{\mathbb{I N}} \forall a^{\beth 2}$ (the application $\quad(x, y) \longmapsto m y+x$ is a bijection

from In onto aIn $\times(1-a) \beth \mathbf{n})$.

from a $\mathbf{m} \times a \rrbracket \mathbf{n}$ onto $a \rrbracket(\mathbf{m n}))$.

Proof.

i) Trivial : the inverse is $\left(y, y^{\prime}\right) \longmapsto y+y^{\prime}$.

ii) By theorem 4.21, this application is injective ; clearly, it sends $a \mathbf{I m} \times a \mathbf{I n}$ into $a \mathbf{I}(\mathbf{m n})$.

Conversely, if $z \varepsilon a \mathbf{I}(\mathbf{m n})$, then there exists $x \varepsilon \mathbf{I m}$ and $y \varepsilon \mathbf{I n}$ such that $z=m y+x$; thus, we have $z=a z=m a y+a x$ with $a x \varepsilon a \beth \mathbf{m}$ and $a y \varepsilon a \mathbf{I n}$. 
Theorem 5.7. We suppose that, for each $\alpha \in \Lambda, \pi \in \Pi$, and every distinct $\zeta_{0}, \zeta_{1}, \zeta_{2} \in \Lambda$, we have $k_{\pi} \alpha \zeta_{0} \Vdash \perp$ or $k_{\pi} \alpha \zeta_{1} \Vdash \perp$ or $k_{\pi} \alpha \zeta_{2} \Vdash \perp$.

Then, for each formula $F(x, y, z)$ with three free variables, we have :

$\theta \Vdash \forall z \forall m^{\mathbb{I N}} \forall n^{\mathbb{I N}} \forall a^{\mathfrak{I 2}}[(2 m<n)=1 \hookrightarrow$

$\left.\left(a \neq 0, \forall x \forall y \forall y^{\prime}\left(F(x, y, z), F\left(x, y^{\prime}, z\right), y \neq y^{\prime} \rightarrow \perp\right), \forall y^{\operatorname{In}} \exists x^{\operatorname{Im}} F(x, a y, z) \rightarrow \perp\right)\right]$

with $\theta=\lambda a \lambda x \lambda y(\mathrm{cc}) \lambda k(y) \lambda z(x z z)(k) a z$.

Remark. This formula means that, if $n>2 m, a \varepsilon \mathbf{I}, a \neq 0$, then there is no surjection from Im onto $a \beth n$ : it suffices to take $F(x, y, z) \equiv<x, y>\varepsilon z$.

Proof. Reasoning by contradiction, let us consider $m, n \in \mathbb{N}$ with $n>2 m, a \in\{0,1\}$, an individual $c$, three terms $\alpha, \xi, \eta \in \Lambda$ and $\pi \in \Pi$ such that :

$\theta \star \alpha \cdot \xi \cdot \eta \cdot \pi \notin \Perp, \quad \alpha \Vdash a \neq 0, \quad \xi \Vdash \forall x \forall y \forall y^{\prime}\left(F(x, y, c), F\left(x, y^{\prime}, c\right), y \neq y^{\prime} \rightarrow \perp\right)$, $\eta \Vdash \forall y^{\beth \mathbf{n}} \neg \forall x^{\beth \mathbf{m}} \neg F(x, a y, c)$.

We have $\theta \star \alpha \cdot \xi \cdot \eta \cdot \pi \succ \eta \star \theta^{\prime} \cdot \pi$ and therefore $\eta \star \theta^{\prime} \cdot \pi \notin \Perp$ with $\theta^{\prime}=\lambda z(\xi z z)\left(\mathbf{k}_{\pi}\right) \alpha z$. It follows that, for every $y \in\{0, \ldots, n-1\}$, we have $\theta^{\prime} \nVdash \forall x^{I m} \neg F(x, a y, c)$.

Thus, there exist two functions $y \longmapsto x_{y}$ (resp. $y \longmapsto \zeta_{y}$ ) from $\{0, \ldots, n-1\}$ into $\{0, \ldots, m-1\} \quad\left(\right.$ resp. into $\Lambda$ ), such that $\zeta_{y} \Vdash F\left(x_{y}, a y, c\right)$ and $\theta^{\prime} \star \zeta_{y} \cdot \varpi_{y} \notin \Perp$ (for some suitable stacks $\varpi_{y}$ ).

Now, we have $\theta^{\prime} \star \zeta_{y} \cdot \varpi_{y} \succ \xi \star \zeta_{y} \cdot \zeta_{y} \cdot \kappa_{y} \cdot \varpi_{y}$ with $\kappa_{y}=\mathrm{k}_{\pi} \alpha \zeta_{y}$; therefore, we have : $\xi \star \zeta_{y} \cdot \zeta_{y} \cdot \kappa_{y} \cdot \varpi_{y} \notin \Perp$ for each $y \in\{0, \ldots, n-1\}$.

By hypothesis on $\xi$ (with $y=y^{\prime}$ ), it follows that $\kappa_{y} \nVdash \perp$ for every $y<n$.

It follows first that $\alpha \nVdash \perp$ and therefore, we have $a=1$; thus $\zeta_{y} \Vdash F\left(x_{y}, y, c\right)$.

Moreover, since $n>2 m$, there exist $y_{0}, y_{1}, y_{2}<n$ distinct, such that $x_{y_{0}}=x_{y_{1}}=x_{y_{2}}$.

But, following the hypothesis of the theorem, the terms $\zeta_{y_{0}}, \zeta_{y_{1}}, \zeta_{y_{2}}$ cannot be distinct, because $\kappa_{y_{0}}, \kappa_{y_{1}}, \kappa_{y_{2}} \nVdash \leftarrow \perp$. Therefore we have, for instance, $\zeta_{y_{0}}=\zeta_{y_{1}} ;$ then, we apply the hypothesis on $\xi$ with $y=y_{0}, y^{\prime}=y_{1}$, which gives $\xi \star \zeta_{y_{0}} \cdot \zeta_{y_{1}} \cdot \kappa \cdot \varpi \in \Perp$ for every $\kappa \in \Lambda$ and $\varpi \in \Pi$. But it follows that $\xi \star \zeta_{y_{0}} \cdot \zeta_{y_{0}} \cdot \kappa_{y_{0}} \cdot \varpi_{y_{0}} \in \Perp$ which is a contradiction.

Corollary 5.8. With the hypothesis of theorem 5.7, the following formulas are realized:

i) $\forall n^{\widetilde{\mathbb{N}}} \forall a^{\mathbf{I 2}}(a \neq 0 \rightarrow$ there is no surjection from In onto $a \mathbf{I}(\mathbf{n}+\mathbf{1}))$.

ii) $\forall n^{\widetilde{\mathbb{N}}} \forall a^{\beth \mathbf{2}} \forall b^{\beth \mathbf{2}}(a \wedge b=0, b \neq 0 \rightarrow$ there is no surjection from a】n onto $b \mathbf{\beth 2})$.

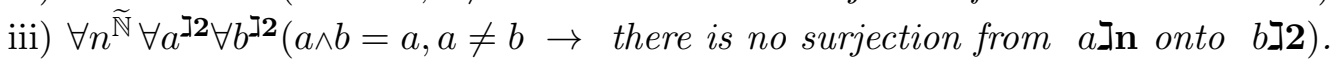

Proof.

i) Suppose that there is a surjection from In onto $a \mathbf{I}(\mathbf{n}+\mathbf{1})$. Then, by the recurrence scheme (theorem $4.12($ ii) ), we see that, for each integer $k \in \widetilde{\mathbb{N}}$, there exists a surjection from $(\mathbf{I n})^{k}$ onto $(a \Xi(\mathbf{n}+\mathbf{1}))^{k}$; and, by proposition 5.6(ii) and the recurrence scheme, it follows that there is a surjection from $\mathbf{I}\left(\mathbf{n}^{\mathbf{k}}\right)$ onto $a \mathbf{I}\left((\mathbf{n}+\mathbf{1})^{\mathbf{k}}\right)$.

But, for $k>n$, we have $(n+1)^{k}>2 n^{k}$ and this contradicts theorem 5.7.

ii) Since $a \wedge b=0$, the rings $(a+b)$ In and $a \mathbf{I n} \times b \mathbf{I n}$ are isomorphic. Reasoning by contradiction, there would exist a surjection from $(a+b) \mathbf{I n}$ onto $b \mathbf{I} \mathbf{2} \times b \mathbf{I n}$, thus also onto $b \mathbf{I}(\mathbf{2 n})$ (proposition 5.6(ii)), thus a surjection from In onto $b \mathbf{I}(\mathbf{2 n})$, which contradicts (i). iii) Otherwise, there would exist a surjection from $a \rrbracket \mathbf{n}$ onto $(b-a) \mathbf{I 2}$, which contradicts (ii).

\section{Applications.}

i) By DC, since $\mathbf{I 2}$ is atomless, there exists in $\mathbf{I} \mathbf{2}$ a strictly decreasing sequence. Hence, by 
corollary 5.8 (iii) and theorem 4.23 , there exists a sequence of infinite subsets of $\mathcal{P}(\widetilde{\mathbb{N}})$, the "cardinals" of which are strictly decreasing.

ii) Applying corollary 5.8 (ii) with $n=2$, we see that there exist two subsets of $\mathcal{P}(\widetilde{\mathbb{N}})$ the "cardinals" of which are incomparable; which means that there is no surjection of one of them onto the other.

More precisely, let $\mathcal{B}$ be the image of $\mathbf{\beth 2}$ by the injection in $\mathcal{P}(\widetilde{\mathbb{N}})$ given by theorem 4.23 ; then we have :

Theorem 5.9. With the hypothesis of theorem 5.7, the following formula is realized in $\mathcal{N}$ : "There exists a subset $\mathcal{B}$ of $\mathcal{P}(\widetilde{\mathbb{N}})$ (the real line of the model $\mathcal{N})$, such that

$\mathcal{B}$ is an atomless Boolean algebra for the usual order $\subseteq$ on $\mathcal{P}(\widetilde{\mathbb{N}})$, with $\emptyset, \widetilde{\mathbb{N}} \in \mathcal{B}$; $a, b \in \mathcal{B} \Rightarrow a \cap b \in \mathcal{B}$.

If $a \in \mathcal{B}, a \neq \emptyset$ then $a \mathcal{B}$ is infinite and there is no surjection from $\mathcal{B}$ onto a $\mathcal{B} \times a \mathcal{B}$ (where aB means $\{x \in \mathcal{B} ; x \subseteq a\}$ ).

If $a, b \in \mathcal{B}, a, b \neq \emptyset$ and $a \cap \bar{b}=\emptyset$, then there is no surjection from a $\mathcal{B}$ onto $b \mathcal{B}$ (the "cardinals" of $a \mathcal{B}, b \mathcal{B}$ are incomparable).

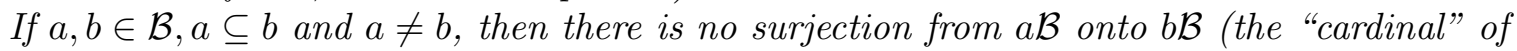
$a \mathcal{B}$ is strictly less than the "cardinal" of $b \mathcal{B})$ ".

In other words, for $a, b \in \mathcal{B}$, we have $: a \subseteq b \Leftrightarrow$ there exists a surjection from $b \mathcal{B}$ onto $a \mathcal{B}$. The order, in the atomless Boolean algebra $\mathcal{B}$, is the order on the "cardinals" of its initial segments.

The model of threads. This model is the canonical instance of a non trivial coherent realizability model. It is defined as follows :

Let $n \longmapsto \pi_{n}$ be an enumeration of the stack constants and let $n \longmapsto \theta_{n}$ be a recursive enumeration of the proof-like terms. For each $n \in \mathbb{N}$, the thread with number $n$ is the set of processes which appear during the execution of the process $\theta_{n} \star \pi_{n}$. In other words, it is the set of all processes $\xi \star \pi$ such that $\theta_{n} \star \pi_{n} \succ \xi \star \pi$.

Note that every term which appears in the $n$-th thread contains the only stack constant $\pi_{n}$. We define $\Perp^{c}$ (the complement of $\Perp$ ) as the union of all threads. Thus, a process $\xi \star \pi$ is in $\Perp^{c}$ iff $(\exists n \in \mathbb{N}) \theta_{n} \star \pi_{n} \succ \xi \star \pi$.

Therefore, we have $\xi \star \pi \in \Perp$ iff the process $\xi \star \pi$ never appears in any thread.

For every term $\xi$, we have $\xi \Vdash \perp$ iff $\xi$ never appears in head position in any thread.

If $\xi$ is a proof-like term, we have $\xi=\theta_{n}$ for some integer $n$, and therefore $\xi \star \pi_{n} \notin \Perp$, by definition of $\Perp$. It follows that the model of threads is coherent.

If $\xi \in \Lambda, \xi \nVdash \perp$ then $\xi$ appears in head position in at least one thread. This thread is unique, unless $\xi$ is a proof-like term, because it is determined by the number of any stack constant which appears in $\xi$.

Theorem 5.10. The hypothesis of theorems 5.1, 5.2, 5.3 and 5.7 are satisfied in the model of threads.

Proof. The hypothesis of theorems 5.3 and 5.1 are trivially satisfied if we take :

$\omega=(\lambda x x x) \lambda x x x, \quad \omega_{0}=(\omega) \underline{0}$, and $\omega_{1}=(\omega) \underline{1}$.

Moreover, the hypothesis of theorem [5.7 is obviously stronger than the hypothesis of theorem 5.2 . 
We check the hypothesis of theorem 5.7 by contradiction :

Suppose that $\mathrm{k}_{\pi} \alpha \zeta_{0} \nVdash \downarrow \perp, \mathrm{k}_{\pi} \alpha \zeta_{1} \nVdash \leftarrow \perp$ and $\mathrm{k}_{\pi} \alpha \zeta_{2} \nVdash \leftarrow \perp$. Therefore, these three terms appear in head position, and moreover in the same thread : indeed, since they contain the stack $\pi$, this thread has the same number as the stack constant of $\pi$.

Let us consider their first appearance in head position, for instance with the order $0,1,2$. Therefore we have, in this thread : $\mathrm{k}_{\pi} \alpha \zeta_{0} \star \rho_{0} \succ \alpha \star \pi \succ \cdots \succ \mathrm{k}_{\pi} \alpha \zeta_{1} \star \rho_{1} \succ \alpha \star \pi \succ \cdots$ But, at the second appearance of $\alpha \star \pi$, the thread enters into a loop, and the term $\mathrm{k}_{\pi} \alpha \zeta_{2}$ can never arrive in head position, since $\zeta_{1} \neq \zeta_{2}$.

\section{REFERENCES}

[1] S. Berardi, M. Bezem, T. Coquand. On the computational content of the axiom of choice. J. Symb. Log. 63 (1998), p. 600-622.

[2] H.B. Curry, R. Feys. Combinatory Logic. North-Holland (1958).

[3] W. Easton. Powers of regular cardinals. Ann. Math. Logic 1 (1970), p. 139-178.

[4] H. Friedman. The consistency of classical set theory relative to a set theory with intuitionistic logic. Journal of Symb. Logic, 38 (2) (1973) p. 315-319.

[5] H. Friedman. Classically and intuitionistically provably recursive functions. In: Higher set theory. Springer Lect. Notes in Math. 669 (1977) p. 21-27.

[6] J.Y. Girard. Une extension de l'interprétation fonctionnelle de Gödel à l'analyse. Proc. 2nd Scand. Log. Symp. (North-Holland) (1971) p. 63-92.

[7] T. Griffin. A formula-as-type notion of control. Conf. record 17th A.C.M. Symp. on Principles of Progr. Languages (1990).

[8] S. Grigorieff. Combinatorics on ideals and forcing. Ann. Math. Logic 3(4) (1971), p. 363-394.

[9] W. Howard. The formulas-as-types notion of construction. Essays on combinatory logic, $\lambda$-calculus, and formalism, J.P. Seldin and J.R. Hindley ed., Acad. Press (1980) p. 479-490.

[10] J. M. E. Hyland. The effective topos.

The L.E.J. Brouwer Centenary Symposium (Noordwijkerhout, 1981), 165-216, Stud. Logic Foundations Math., 110, North-Holland, Amsterdam-New York, 1982.

[11] G. Kreisel. On the interpretation of non-finitist proofs $I$. J. Symb. Log. 16 (1951) p. 248-26.

[12] G. Kreisel. On the interpretation of non-finitist proofs II. J. Symb. Log. 17 (1952), p. 43-58.

[13] J.-L. Krivine. Typed lambda-calculus in classical Zermelo-Fraenkel set theory. Arch. Math. Log., 40, 3, p. 189-205 (2001). http://www.pps.jussieu.fr/ krivine/articles/zf_epsi.pdf

[14] J.-L. Krivine. Dependent choice, 'quote' and the clock. Th. Comp. Sc., 308, p. 259-276 (2003). http://hal.archives-ouvertes.fr/hal-00154478 Updated version at :

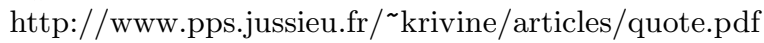

[15] J.-L. Krivine. Realizability in classical logic.

In Interactive models of computation and program behaviour. Panoramas et synthèses, Société Mathématique de France, 27, p. 197-229 (2009). http://hal.archives-ouvertes.fr/hal-00154500 Updated version at :

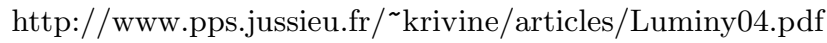

[16] J.-L. Krivine. Realizability : a machine for Analysis and set theory. Geocal'06 (febr. 2006 - Marseille); Mathlogaps'07 (june 2007 - Aussois). http://cel.archives-ouvertes.fr/cel-00154509 
Updated version at :

http://www.pps.jussieu.fr/ krivine/articles/Mathlog07.pdf

[17] J.-L. Krivine. Structures de réalisabilité, RAM et ultrafiltre sur $\mathbb{N}$. (2008)

http://hal.archives-ouvertes.fr/hal-00321410

Updated version at :

http://www.pps.jussieu.fr/ krivine/articles/Ultrafiltre.pdf

[18] J.-L. Krivine. Realizability algebras : a program to well order $\mathbb{R}$.

Logical Methods in Computer Science, vol. 7, 3:02, p. 1-47 (2011)

http://hal.archives-ouvertes.fr/hal-00483232

Updated version at :

http://www.pps.jussieu.fr/ krivine/articles/Well_order.pdf 